\title{
Tubular Dickkopf-3 promotes the development of renal atrophy and fibrosis
}

\author{
Giuseppina Federico, ${ }^{1}$ Michael Meister, ${ }^{2}$ Daniel Mathow, ${ }^{1}$ Gunnar H. Heine, ${ }^{3}$ Gerhard Moldenhauer, ${ }^{2}$ \\ Zoran V. Popovic, ${ }^{1}$ Viola Nordström, ${ }^{1}$ Annette Kopp-Schneider, ${ }^{4}$ Thomas Hielscher, ${ }^{4}$ \\ Peter J. Nelson, ${ }^{5}$ Franz Schaefer, ${ }^{6}$ Stefan Porubsky, ${ }^{1}$ Danilo Fliser, ${ }^{3}$ Bernd Arnold, ${ }^{2}$ \\ and Hermann-Josef Gröne ${ }^{1}$ \\ 'Department of Cellular and Molecular Pathology and 'Department of Molecular Immunology, German Cancer Research \\ Center, Heidelberg, Germany. ${ }^{3}$ Department of Internal Medicine IV, Nephrology and Hypertension, Saarland University \\ Medical Center, Homburg, Germany. ${ }^{4}$ Department of Biostatistics, German Cancer Research Center, Heidelberg, Germany. \\ ${ }^{5}$ Clinical Biochemistry Group, Department of Internal Medicine and Policlinic IV, Ludwig-Maximilians-University (LMU), \\ Munich, Germany. ${ }^{6}$ Division of Pediatric Nephrology, University of Heidelberg, Heidelberg, Germany.
}

Renal tubular atrophy and interstitial fibrosis are common hallmarks of etiologically different progressive chronic kidney diseases (CKD) that eventually result in organ failure. Even though these pathological manifestations constitute a major public health problem, diagnostic tests, as well as therapeutic options, are currently limited. Members of the dickkopf (DKK) family, DKK1 and -2 , have been associated with inhibition of Wnt signaling and organ fibrosis. Here, we identify DKK3 as a stress-induced, tubular epithelia-derived, secreted glycoprotein that mediates kidney fibrosis. Genetic as well as antibody-mediated abrogation of DKK3 led to reduced tubular atrophy and decreased interstitial matrix accumulation in two mouse models of renal fibrosis. This was facilitated by an amplified, antifibrogenic, inflammatory $T$ cell response and diminished canonical $W n t / \beta$-catenin signaling in stressed tubular epithelial cells. Moreover, in humans, urinary DKK3 levels specifically correlated with the extent of tubular atrophy and interstitial fibrosis in different glomerular and tubulointerstitial diseases. In summary, our data suggest that DKK3 constitutes an immunosuppressive and a profibrotic epithelial protein that might serve as a potential therapeutic target and diagnostic marker in renal fibrosis.

Authorship note: G. Federico and M. Meister contributed equally to this work. B. Arnold and H.-J. Gröne are co-senior authors.

\section{Conflict of interest: A patent} application has been deposited by H.-J. Gröne and B. Arnold for DKK3 as a urinary biomarker in CKD.

Submitted: December 3, 2015 Accepted: December 17, 2015 Published: January 21, 2016

Reference information: JCI Insight. 2016;1(1):e84916. doi:10.1172/ji.insight.84916.

\section{Introduction}

Tubular atrophy (TA) and interstitial fibrosis (IF) are the relevant denominators of chronic kidney diseases (CKD). CKD represent a major public health issue with limited diagnostic and therapeutic options. Renal fibrogenesis is considered to be a failed wound-healing process that occurs with persistent injurious insults (1). Development of renal fibrosis is thought to be facilitated by the induction of a nonresolving inflammation that drives the activation and expansion of matrix-producing fibroblasts, resulting in an accumulation of extracellular matrix (ECM) proteins $(2,3)$. Besides being a target of fibrosis, tubular epithelial cells (TECs) play an essential role in the orchestration of renal fibrosis, predominantly through crosstalk with other cell types via growth factors, cytokines, and chemokines. Upon activation and injury, TECs were shown to secrete connective tissue growth factor (CTGF) - a key mediator of tissue fibrosis (4) and cytokines such as IL-6, IL-8, and TNF (5) - as well as CCL5 and monocyte chemoattractant protein-1 (MCP1) (6-8). The cytokines regulate the recruitment and activation of $\mathrm{T}$ cells and macrophages that represent major hematopoietic effector cells for renal fibrosis $(9,10)$. The signaling pathways and transcriptional programs that regulate the activity of TECs in fibrogenesis have not been defined in detail.

Dickkopf (DKK) proteins constitute an evolutionarily conserved family that consists of five secreted glycoproteins: DKK1-4, which share two conserved cysteine-rich domains (CRDs), and a divergent member, soggy (11). The N-terminal CRD is unique to the DKK family and is not found in other vertebrate proteins. The two CRDs are separated by a linker region, similar in DKK1, -2 , and -4 but significantly shorter in DKK3 (12). During embryogenesis, DKK proteins are coordinately expressed in mesenchymal lineages (13), which also give rise to kidney development (14). 
DKK family members have been reported to be modulators of Wnt/ $\beta$-catenin pathways, which are thought to play a significant role in renal development and disease (15-17). While DKK1, -2 , and -4 have been demonstrated to directly interact with the Wnt/ $\beta$-catenin pathways (18-20), DKK3 has not unequivocally been associated with Wnt signaling (21). Some publications assert that DKK3 inhibits, while others state that DKK3 potentiates Wnt signaling, depending on the cellular context (22-24). In recent reports, different proteins of the Wnt signaling cascade have been suggested to interact with DKK3 $(25,26)$.

We have recently demonstrated that DKK3 can act as a tissue-derived immune modulator that influences type and strength of local $\mathrm{T}$ cell responses in models of peripheral tolerance, transplantation, and autoimmune disease (27-29). We have found that DKK3 is expressed during kidney development, which occurs by a mesenchymal-epithelial transition. As DKK3 can function as an immune modulator and is expressed in mesenchyme-derived tissue, we hypothesized that DKK3 can influence chronic inflammatory fibrosing kidney disease.

In the present study, we uncovered that stress-induced TEC-derived DKK3 is a driver of renal fibrosis. Intriguingly, DKK3 mediated an immunosuppressive and, at the same time, a profibrotic function by interaction with Wnt signaling. Genetic abrogation as well as antibody-mediated blockade of DKK3 markedly reduced kidney damage and improved renal function in two different models of renal atrophy and fibrosis. We also identified urinary DKK3 as a noninvasivediagnostic marker that identifies the degree of atrophy and fibrosis in human patients with different types of CKD.

\section{Results}

$D K K 3$ promotes $T A$ and IF. In order to investigate the role of DKK3 in CKD, we analyzed the phenotype of DKK3-deficient $\left(D k k 3^{---}\right)$mice in a well-characterized mouse model of renal fibrosis: unilateral ureteral obstruction (UUO). Morphometric evaluation and keratin-18 immunofluorescence staining revealed only minor phenotypic alterations 7 days after UUO. However, 21 days after obstruction, we detected considerably diminished TA and dedifferentiation in $D k k 3^{-1-}$ mice compared with littermate controls (Figure 1, A-C). Moreover, trichrome staining and fibronectin-immunolabeling displayed a significant reduction of interstitial ECM deposition in kidneys of these mice (Figure 1, D-F). Accordingly, accumulation of $\alpha$ smooth muscle actin-positive ( $\alpha$-SMA-positive) myofibroblasts was also diminished in $D k k 3^{-1-}$ mice (Supplemental Figure 1; supplemental material available online with this article; doi:10.1172/jci.insight.84916DS1). As DKK3 was shown to modulate local $\mathrm{T}$ cell responses (27), we evaluated whether it may influence the inflammatory response that drives fibrogenesis in the kidney. Surprisingly, even though DKK3 deficiency led to significantly diminished renal pathology, we detected increased numbers of CD3-positive $\mathrm{T}$ cells within kidneys of $D k k 3^{-1-}$ mice 7 and 21 days after UUO (Figure 1, G and H). In contrast, numbers of F4/80-positive monocytes were comparable in obstructed kidneys of both strains (Supplemental Figure 2A). A detailed analysis of the T cell infiltrate 21 days after UUO revealed increased levels of IFN $\gamma$ - and

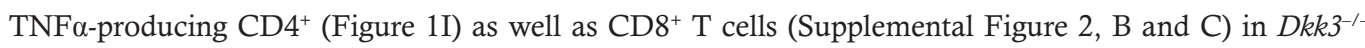
samples. At the same time, we found fewer GATA3-positive CD4 ${ }^{+} \mathrm{T}$ cells (Figure $1 \mathrm{~J}$ ) but increased numbers and proportions of $\mathrm{CD} 4{ }^{+} \mathrm{CD} 25^{+} \mathrm{FOXP} 3^{+}$Tregs, which apparently prevented uncontrolled $\mathrm{Th} 1 \mathrm{~T}$ cell response (Figure 2, $\mathrm{K}$ and $\mathrm{L}$ ). In contrast to the obstructed kidneys, alteration of the $\mathrm{T}$ cell phenotypes was not apparent in the respective spleens (Supplemental Figure 3). Thus, these data suggest that DKK3 locally, in the renal microenvironment, regulates the degree and type of the adaptive immune response and promotes the development of TA and IF.

Antibody-mediated DKK3 blockade ameliorates renal fibrosis. In order to assess whether direct blockade of DKK3 protein could corroborate the beneficial effect of the genetic DKK3 abrogation and to investigate the therapeutic potential of antibody-mediated DKK3 blockade, we treated WT UUO mice with either an anti-DKK3 monoclonal antibody or an isotype control. The specificity of the DKK3 antibody has been shown in experiments in which anti-DKK3 selectively targeted regulatory CD8 ${ }^{+} \mathrm{T}$ cells positive for DKK3 (28). The first antibody injection was done at surgery, followed by applications on every third day (Figure 2A). Twenty-one days after obstruction, anti-DKK3-treated mice displayed less TA (Figure 2, B and C), diminished IF (Figure 2, D and E), and increased $\mathrm{T}$ cell numbers within the kidney (Figure 2F), comparable to those caused by genetic deletion of $D k k 3$. In summary, these results show that the genetic DKK3 model is not influenced by unknown compensatory mechanisms and that DKK3 represents a potential therapeutic target to treat renal TA and fibrosis.

Profibrogenic DKK3 function in the kidney is independent of the cause of initial damage. To demonstrate that 
A

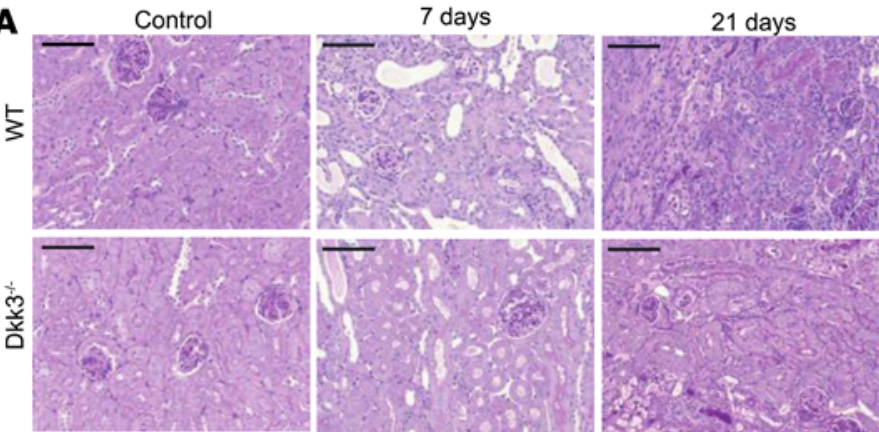

D

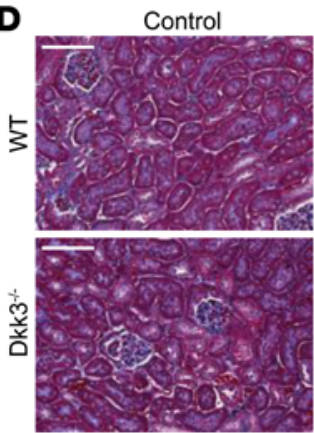

G

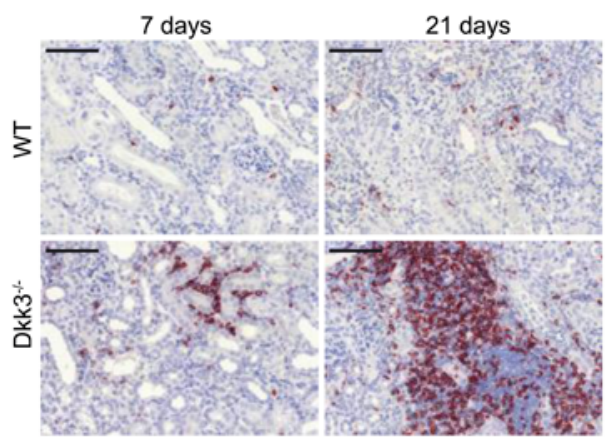

J

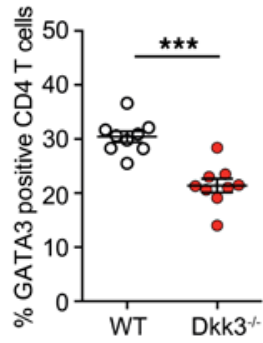

7 days
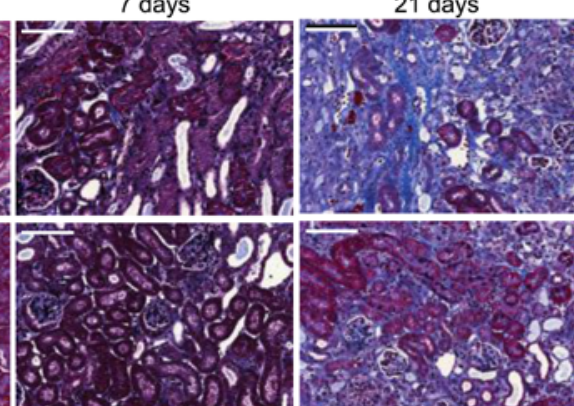

H
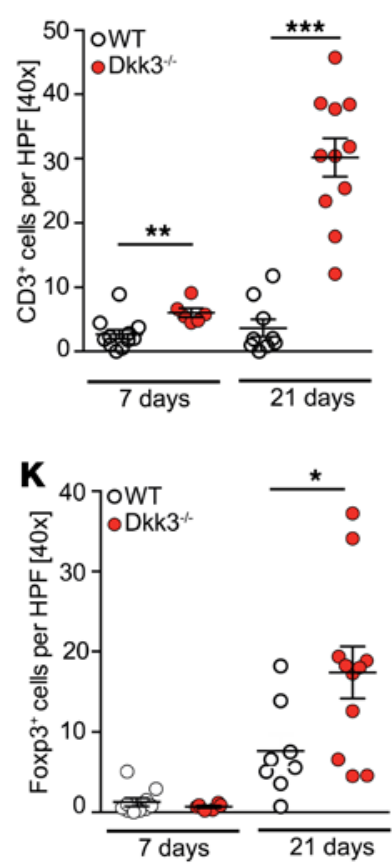

B

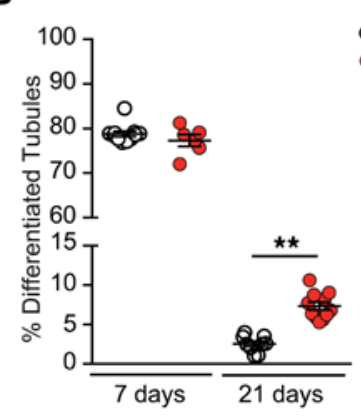

E

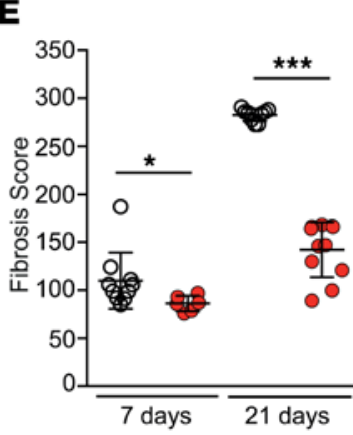

C DAPI Keratin18

OWT -Dkk3*

5

旁

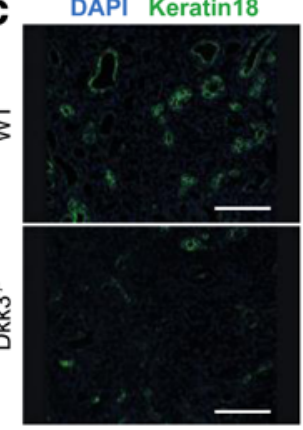

F DAPI Fibronectin 乡

旁
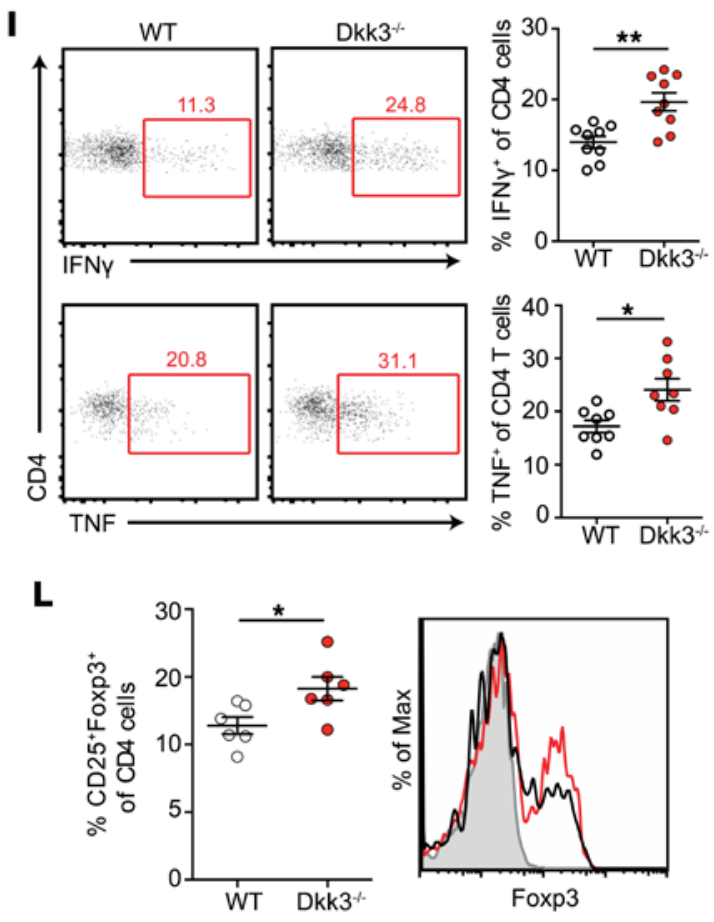
Figure 1. Dickkopf 3 (DKK3) abrogation diminishes tubular damage and renal interstitial fibrosis accompanied by an amplified, Th1-skewed T cell response in the obstructed kidney. (A) Representative images of Periodic acid-Schiff-stained (PAS-stained) kidney sections of WT ( $n=11 / 9$ ) and $D k k 3^{-1-}$ $(n=6 / 11)$ mice 0,7 , and 21 days after unilateral ureteral obstruction (UUO) (scale bars: $100 \mu \mathrm{m}$ ). (B) Quantification of segmental differentiated tubules in PAS-stained kidney sections of WT $(n=11 / 9)$ and $D \mathrm{kk3}^{-1-}(n=6 / 11)$ mice 0 (control), 7, and 21 days after UUO. (C) Representative keratin-18 immunofluorescence images of kidney sections from WT $(n=3)$ and $D k_{k} 3^{-1-}(n=3)$ mice 21 days after UUO. Nuclei were stained with DAPI (scale bars: $\left.100 \mu \mathrm{m}\right)$. (D) Representative images of Masson's trichrome-stained kidney sections of WT $(n=11 / 9)$ and $D k k 3^{-1-}(n=6 / 11)$ mice 0,7 , and 21 days after UUO (scale bars: $100 \mu \mathrm{m})$. (E) Semiquantitative analysis of renal interstitial fibrosis in Masson's trichrome-stained kidney sections of WT $(n=11 / 9)$ and $D k k^{-/-}(n=6 / 11)$ mice 0 (control), 7, and 21 days after UUO. (F) Representative fibronectin immunofluorescence images of kidney sections from WT $(n=3)$ and $D k k 3^{-1-}(n=3)$ mice 21 days after UUO. Nuclei were stained with DAPI (scale bars: $100 \mu \mathrm{m})$. (G) Representative CD3 IHC in kidneys of WT $(n=11 / 9)$ and $D \mathrm{kk} 3^{-1-}(n=6 / 11)$ mice 7 and 21 days after UUO (scale bars: $100 \mu \mathrm{m})$. (H) Quantification of $\mathrm{CD3}^{+}$cells per high-power field $(\times 40)$ in WT $(n=11 / 9)$ and $D k k 3^{-/-}(n=6 / 11)$ mouse kidneys 7 and 21 days after UUO. (I) Flow cytometric analysis of T cells, isolated from WT and $D k k 3^{-/-}$mouse kidneys 21 days after UUO. Representative dot plots of IFN $\gamma$ - and TNF $\alpha$-producing CD4+ cells (left panel). Percentages of IFN $\gamma$ - and TNF $\alpha$-producing CD4 ${ }^{+}$cells (right panel) $(n=8 / 9)$. (J) Percentage of GATA3 ${ }^{+}$of CD4 ${ }^{+}$cells (left panel) $(n=9 / 9)$ and the related GATA3 expression (right panel) analyzed by flow cytometry. (K) Quantification of FoxP3 ${ }^{+}$cells per high-power field $(\times 40)$ in kidney slides of WT and $D k k 3^{-/-}$mice $7(n=10 / 6)$ and 21 days $(n=8 / 11)$ after UUO. (L) Percentage of CD25+FoxP3 $3^{+}$of CD4 $4^{+}$cells (left panel) $(n=6 / 6)$ and the respective FoxP3 histogram (right panel), analyzed by flow cytometry. All data are shown as mean \pm SEM. Statistical analysis performed using either Mann-Whitney $U$ test (B, E, $\mathbf{H}$, and $\mathbf{K}$ ) or an unpaired, 2-tailed $t$ test $(\mathbf{I}, \mathbf{J}$, and $\mathbf{L}) .{ }^{*} P<0.05 ;{ }^{* *} P<0.01 ;{ }^{* *} P<0.001$.

the profibrotic DKK3 function in the kidney is not restricted to a mouse model with a physical mechanism of injury (obstruction of urine flow), we made use of a second mouse model, adenine nephropathy. In parallel to the UUO model, less tubular damage (Figure 3, A-C) as well as reduced IF (Figure 3, D-F, and Supplemental Figure 4) was detected in DKK3-deficient animals after 28 days of an adenine-rich diet. In addition, reduced plasma creatinine levels revealed preserved renal function in $D k k 3^{-1-}$ mice upon adenine feeding (Figure 3G). Numbers of $\mathrm{CD}^{+} \mathrm{T}$ cells within the respective kidneys were again increased in DKK3-deficient mice after 4 weeks of adenine administration (Figure 3H). Thus, DKK3 seems to function as a profibrotic factor in chronic renal tubulointerstitial disease, irrespective of the underlying etiology.

$T E C$ are the predominant source of profibrotic DKK3 in the kidney. To determine whether the observed phenotype in $D k k^{---}$kidneys was caused by a $\mathrm{T}$ cell-intrinsic defect, isolated $\mathrm{T}$ lymphocytes were adoptively transferred into recombination activating gene $2-$ deficient mice (Rag2-- mice), which lack mature $\mathrm{B}$ and $\mathrm{T}$ cells, two days before generating UUO. Twenty-one days after surgery, injection of either WT or $D k k 3^{---} \mathrm{T}$

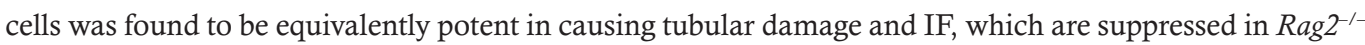
mice without lymphoid cell repletion (Figure $4, \mathrm{~A}-\mathrm{C}$ ). To identify the particular cell type producing DKK3 upon UUO, we generated a BAC-transgenic reporter mouse ( $D k k 3-L C h)$ expressing luciferase and mCherry under the regulatory sequences of the $D k k 3$ gene (Supplemental Figure 5A). Ex vivo bioluminescence imaging of obstructed $D k k 3-L C h$ kidneys revealed an induction of luciferase expression starting 2 days after UUO (Figure 4D). This was confirmed by ELISA measurements, showing increased DKK3 protein levels in whole-organ homogenates of obstructed kidneys compared with untreated controls (Supplemental Figure 5B). Similar results were obtained in the adenine nephropathy model (Supplemental Figure 6, A and B). This stress induction of DKK3 recapitulates DKK3 expression in the developing postnatal mouse kidney (Figure 4E). In order to localize the DKK3-expressing cell type, mCherry expression in Dkk3-LCh mice was analyzed via immunofluorescence microscopy before and 7 days after UUO or at the beginning of an adenine-rich diet. Morphological analysis, as well as costaining with aquaporin 1 (AQP1) for proximal tubules and AQP2 for distal tubules and collecting ducts, revealed an exclusive expression of the reporter gene in stress-activated tubular cells, foremost proximal TECs (Figure $4 \mathrm{~F}$ and Supplemental Figure $6 \mathrm{C}$ ). To investigate whether these cells are indeed the primary source of functional DKK3 within the fibrotic kidney, we generated mice with a conditional DKK3 KO in TECs. Animals containing floxed Dkk3 alleles (Supplemental Figure 7) were crossed to $\mathrm{Pax} 8^{\mathrm{Cr} / \mathrm{+}}$ mice, in which Cre-recombinase expression is specifically directed to all tubular and collecting duct epithelia in kidney (30). Induction of UUO in tubular-specific DKK3 KO mice resulted in decreased TA (Figure 4, G and H) and diminished IF (Figure 4, I and J), similar to the data obtained in global $D k k 3^{-/-}$mice. These data convincingly show that profibrotic DKK3 produced upon injurious stimuli in the kidney is derived from TECs.

DKK3 supports Wht signaling and regulates stress-induced cytokine expression in TECS. To identify global changes in gene expression associated with the lack of DKK3 in the early phase of tubulointerstitial injury, whole-transcriptome sequencing (RNA-seq) was performed on RNA isolated from kidneys of WT and $D k k^{-/-}$mice 7 days after UUO. Gene ontology (GO) analysis indicated major transcriptional changes in immune system-associated pathways. In particular, substantially increased transcriptional activity of genes related to $\mathrm{T}$ cell activation and differentiation, as well as cytokine activity, were 
A

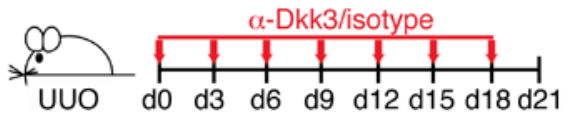

D

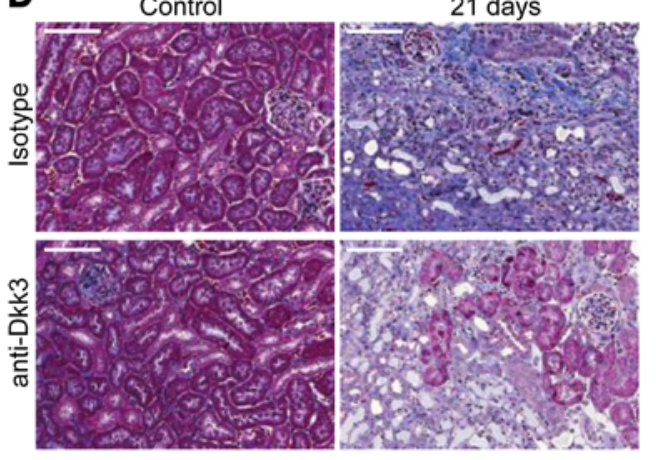

B
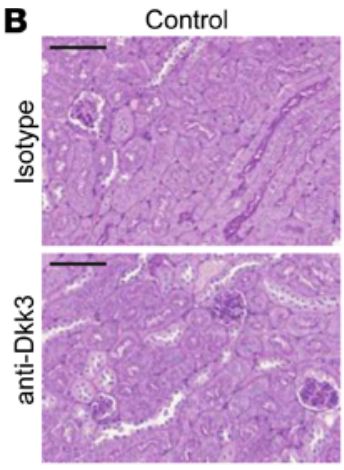

D

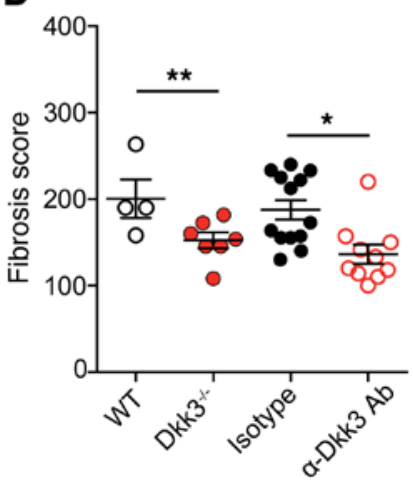

21 days

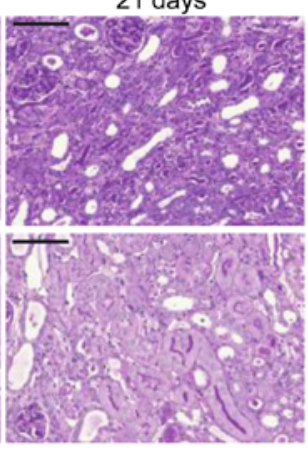

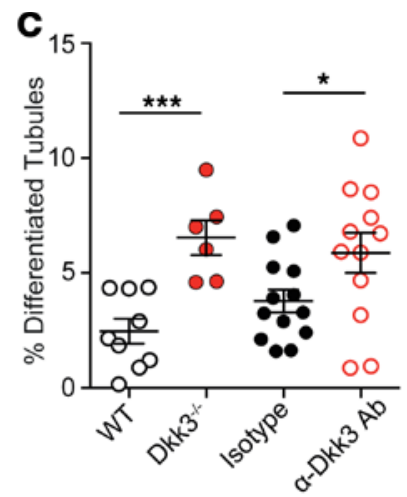

$\mathbf{F}$

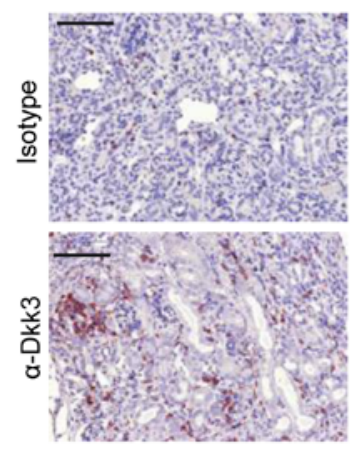

Figure 2. Antibody-mediated dickkopf 3 (DKK3) blockade ameliorates renal tubular damage and interstitial fibrosis. (A) Schematic illustration of the antibody administration protocol. Anti-DKK3 or isotype control antibody $(1 \mathrm{mg})$ was administered to WT mice immediately after unilateral ureteral obstruction (UUO). Afterward, $0.5 \mathrm{mg}$ of the respective antibodies was applied every third day until mice were sacrificed after 21 days. (B) Representative images of Periodic acid-Schiff-stained (PAS-stained) kidney sections from isotype $(n=13)$ and DKK3 antibody-treated $(n=12)$ mice 21 days after UUO (scale bars: $100 \mu \mathrm{m})$. (C) Quantification of segmental differentiated tubules in PAS-stained kidney sections of WT $(n=9), D \mathrm{kk} 3^{--}(n=6)$, isotype- $(n=13)$, and DKK3 antibodytreated $(n=12)$ mice 21 days after UUO. (D) Representative images of Masson's trichrome-stained kidney sections from isotype- $(n=13)$ and DKK3 antibodytreated $(n=12)$ mice 21 days after UUO (scale bars: $100 \mu \mathrm{m}$ ). (E) Semiquantitative analysis of renal interstitial fibrosis in Masson's trichrome-stained kidney sections of WT $(n=9), D k \mathrm{~kJ}^{-1-}(n=6)$, isotype- $(n=13)$, and DKK3 antibody-treated $(n=12)$ mice 21 days after UUO. (F) Representative CD3 IHC of kidneys of DKK3 antibody-injected $(n=6)$ and isotype antibody-injected $(n=6)$ WT mice 21 days after UUO (Scale bars: $100 \mu$ m) (left panel). Quantification of CD3 cells per high power field (HPF, $\times 40$ ) of kidney of DKK3 antibody-injected $(n=6)$ and isotype antibody-injected $(n=6)$ WT mice 21 days after UUO (right panel). All data are shown as mean \pm SEM. Statistical analysis performed using Mann-Whitney $U$ test. ${ }^{*} P<0.05 ;{ }^{* *} P<0.01 ;{ }^{* * *} P<0.001$.

detected in $D k k 3^{---}$kidneys (Figure 5A). Gene expression levels showed preferential Th1 cell differentiation in kidneys of $D k k 3^{--}$mice (Supplemental Figure 8), reflecting the results observed at the cellular level. In addition to immune response-associated genes, RNA-seq data indicated a clear-cut trend in that the ablation of DKK3 induced an alteration of Wnt signaling-related gene expression (Figure 5B). To further study the potential influence of DKK3 on the Wnt/ $\beta$-catenin pathway in situ, in the UUO kidneys, we employed a transgenic mouse line that reports transcriptional $\beta$-catenin activity by nuclear GFP expression under the regulation of the catenin-responsive transcription factors $\mathrm{T}$ cell factor (TCF)/ lymphoid enhancer factor (LEF) (TCF/LEF:H2B-GFP) and crossed these mice to a $D k k 3^{-1-}$ background. While absent in untreated mice, strong induction of nuclear GFP expression was detected in TECs of $T C F / L E F: H 2 B-G F P$ mice 7 days after UUO. In contrast, in DKK3-deficient TCF/LEF:H2B-GFP mice, reporter activity was significantly decreased (Figure 5, C and D). In order to document a direct interaction of DKK3 with Wnt signaling in TECs, a proximity ligation assay was applied in an in vitro cell culture system with human TECs. This assay was set up such that a direct molecular interaction of the cell surface receptor frizzled (FZD) with the cytoplasmic protein disheveled (DVL), occurring upon activating Wnt-ligand (Wnt3a) binding (Supplemental Figure 9A), could be seen. We have found that siRNA-mediated suppression of DKK3 in the human TEC line HK2 (Supplemental Figure 9, B and C) resulted in significantly decreased activation of FZD/DVL interaction upon WNT3a stimulation (Figure 5, E and F). Moreover, we analyzed the impact of siRNA-mediated DKK3 knockdown on the expression of cytokines by tubular cells, which may regulate the activity of interstitial cells. HK2 cells, 
A

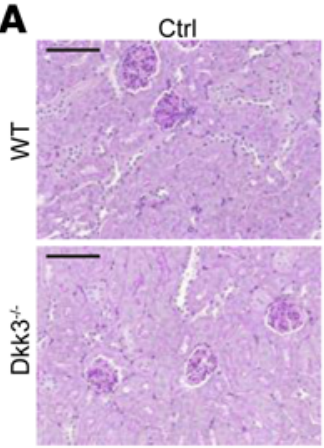

D

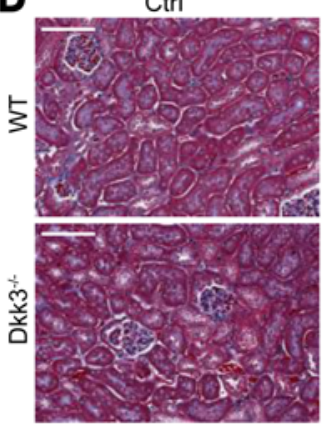

G

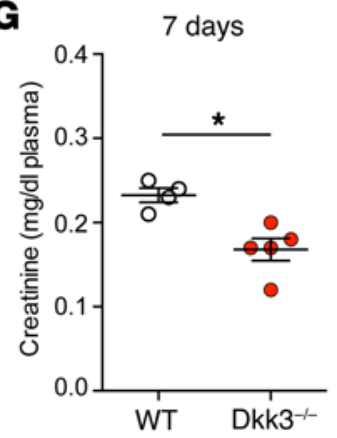

7 days

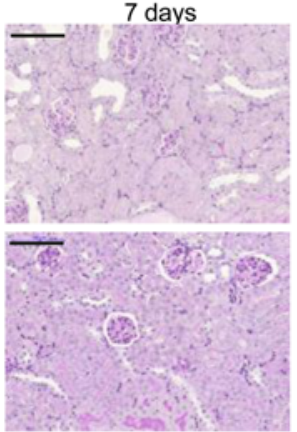

7 days

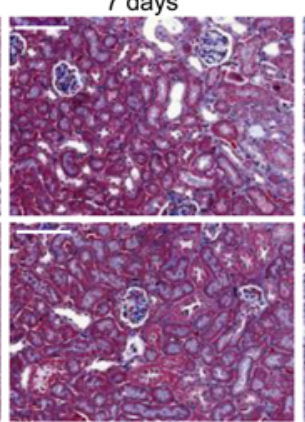

28 days

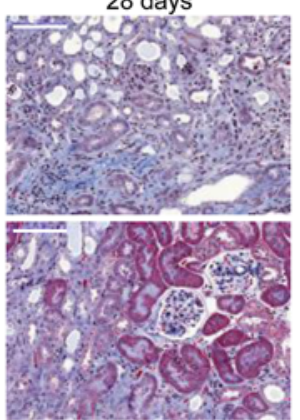

B
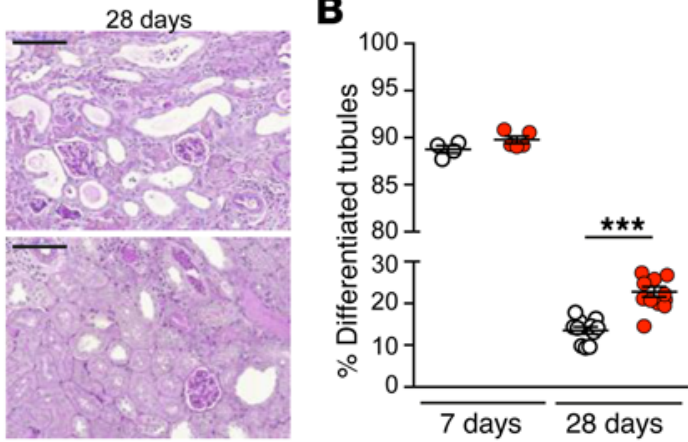

E

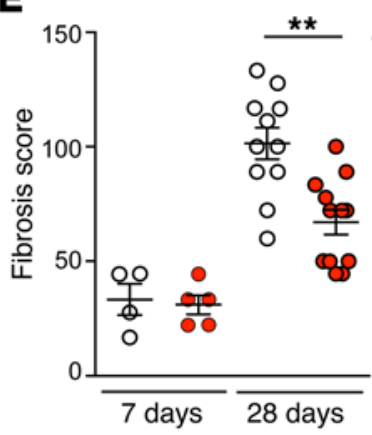

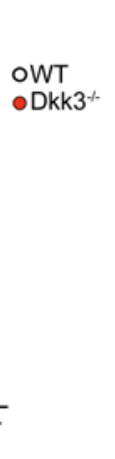

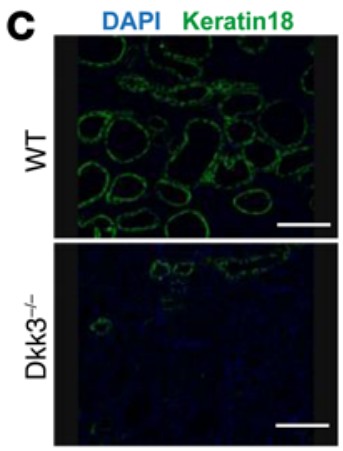

$\mathbf{F}$ OWT -Dkk3

5

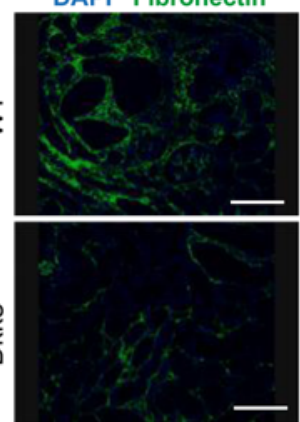

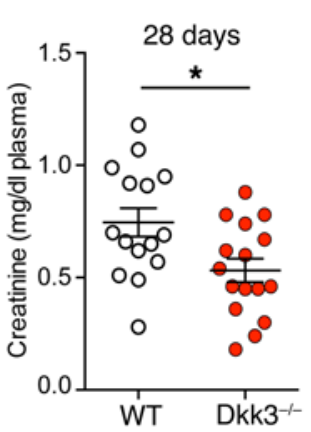
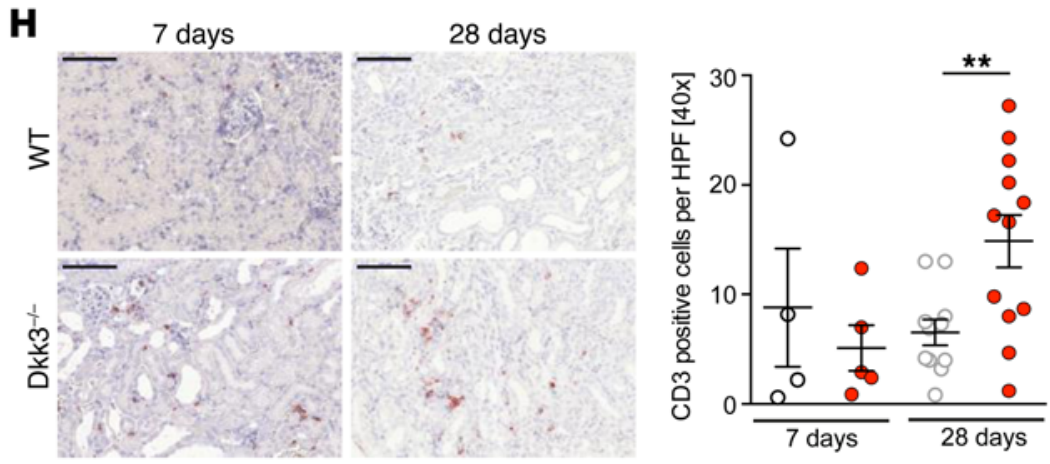

Figure 3. Dickkopf 3 (DKK3) abrogation diminishes tubular damage and renal interstitial fibrosis in the adenine nephropathy model. (A) Representative images of Periodic acid-Schiff-stained (PAS-stained) kidney sections of WT $(n=4 / 9)$ and $D k k 3^{--}(n=5 / 11)$ mice 0,7 , and 28 days after beginning of an adenine-rich diet (scale bars: $100 \mu \mathrm{m}$ ). (B) Quantification of segmentally differentiated tubules in PAS-stained kidney sections of WT ( $n=4 / 9$ ) and $D k k 3^{-}$ ( $n=5 / 11$ ) mice either 7 or 28 days after adenine-rich diet administration. (C) Representative keratin-18 immunofluorescence images of kidney sections of WT $(n=3)$ and $D k k 3^{-1-}(n=3)$ mice 28 days after beginning of an adenine-rich diet (scale bars: $\left.100 \mu \mathrm{m}\right)$. (D) Representative images of Masson's trichromestained kidney sections of WT $(n=4 / 9)$ and $D k k 3^{-/-}(n=5 / 11)$ mice kidney 0,7 , and 28 days after adenine-rich diet administration (scale bars: $\left.100 \mu \mathrm{m}\right)$. (E) Semiquantitative analysis of renal interstitial fibrosis in Masson's trichrome-stained kidney sections of WT $(n=4 / 9)$ and $D k k 3^{-/-}(n=5 / 11)$ mice either 7 or 28 days after beginning of an adenine-rich diet. (F) Representative fibronectin immunofluorescence images of kidney sections of WT ( $n=3$ ) and $D k k 3^{-1-}$ $(n=3)$ mice kidney 28 days after beginning of an adenine-rich diet (scale bars: $100 \mu \mathrm{m})$. (C) Plasma creatinine concentration of WT $(n=4 / 15)$ and $D k k 3^{-/-}$ ( $n=5 / 16)$ mice 7 and 28 days after beginning of an adenine-rich diet. (H) Representative CD3 IHC of WT $(n=11 / 9)$ and $D k k 3^{-1-}(n=6 / 11)$ mouse kidneys 7 and 28 days after beginning of an adenine-rich diet (scale bars: $100 \mu \mathrm{m}$ ) (left panel). Quantification of CD3 ${ }^{+}$cells per high-power field (HPF, $\left.\times 40\right)$ in kidney sections of WT $(n=4 / 11)$ and $D k k 3^{-/-}(n=5 / 12)$ mice 7 and 28 days after beginning of an adenine-rich diet (right panel). All data are shown as mean \pm SEM. Statistical analysis performed using Mann-Whitney $U$ test. ${ }^{*} P<0.05 ;{ }^{* *} P<0.01 ;{ }^{* * *} P<0.001$.

treated with either control or DKK3-siRNA, were exposed to hydrogen peroxide or IFN $\gamma$ as stress triggers. In the supernatant of such cells, increased IL-6 and IL-8 levels were measured in DKK3-siRNAtreated cells compared with controls (Supplemental Figure 10). In summary, our data reveal that DKK3 promotes $\mathrm{Wnt} / \beta$-catenin signaling and regulates cytokine expression in stressed TECs (Figure $5 \mathrm{G}$ ).

Urinary DKK3 correlates with TA and IF in CKD patients. Due to the active production of secreted DKK3 by TECs upon injurious insults, we analyzed DKK3 excretion in the urine of mice before and after beginning an adenine-rich diet via ELISA. While DKK3 protein was not detected in the urine of control mice, progression of kidney damage upon adenine feeding was accompanied by increasing DKK3 concentra- 
A

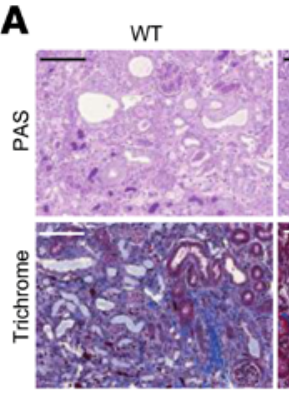

D

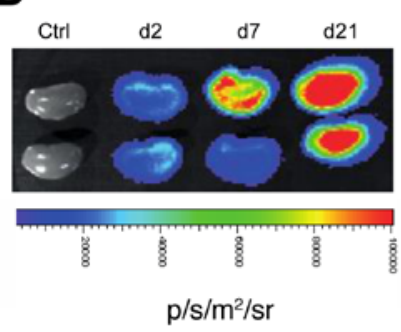

Rag2

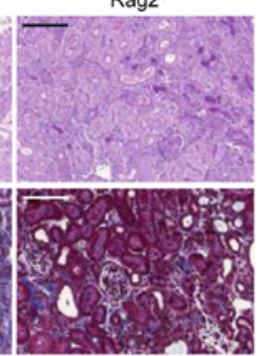

Rag2 ${ }^{*}+$ WT T-cells
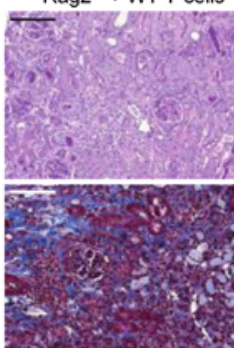

Rag2 + Dkk3* T-cells

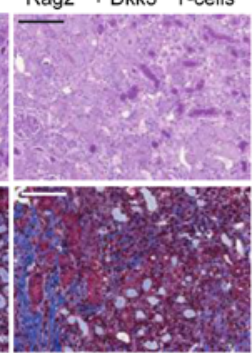

B

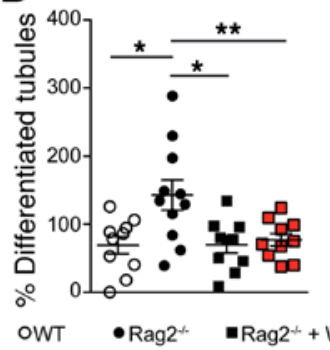

C

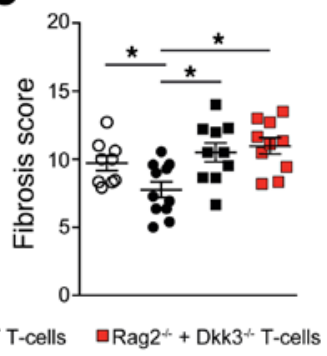

E

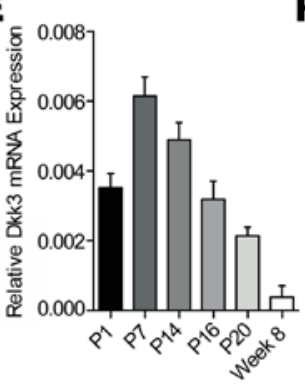

$\mathbf{F}$

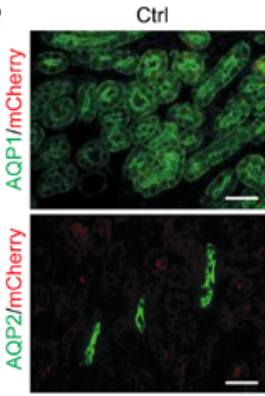

d7

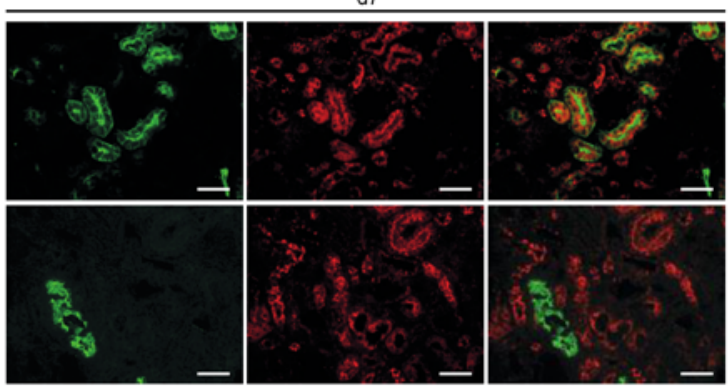

G

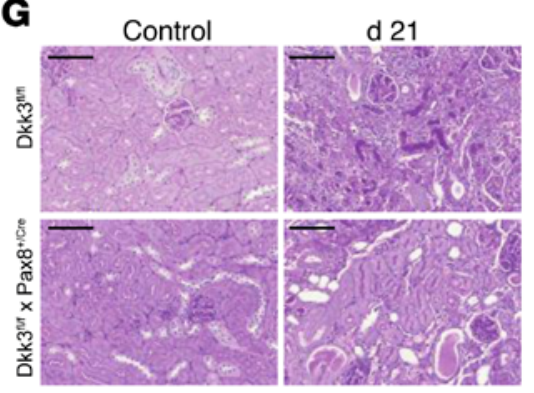

H

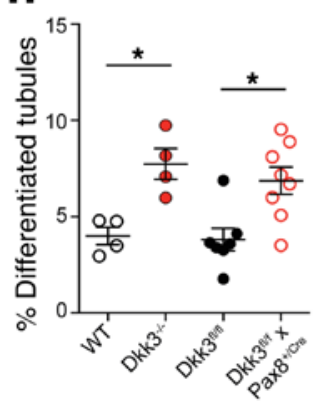

I

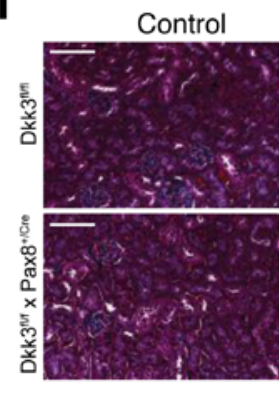

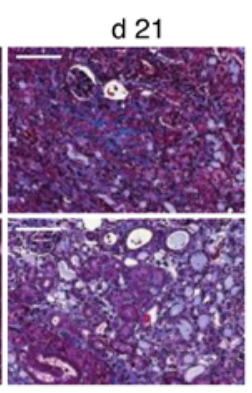

J

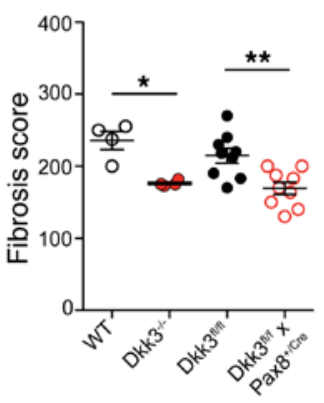

Figure 4. TECs are the predominant source of profibrotic dickkopf 3 (DKK3) in the kidney. (A) Representative images of Periodic acid-Schiff-stained

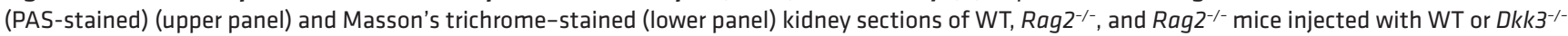
spleen-derived T cells 21 days after unilateral ureteral obstruction (UUO). Each mouse was injected i.p. with $3 \times 10^{7}$ isolated T cells 2 days before surgery (scale bars: $100 \mu \mathrm{m})$. (B) Quantification of renal segmental differentiated tubules in PAS-stained kidney sections of WT $(n=10), \operatorname{Rag} 2^{-/-}(n=11)$, and Rag2 $2^{-/-}$mice injected with WT $(n=10)$ or $D k k^{-/-}$spleen-derived $(n=10)$ T cells 21 days after UUO. (C) Semiquantitative analysis of renal interstitial fibrosis

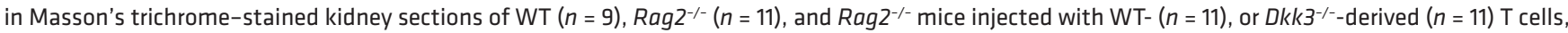
21 days after UUO. (D) Representative ex vivo bioluminescence image of $D k k 3-L C h$ mouse kidneys $0,2,7$, and 21 days after UUO ( $n=3$ ). (E) Relative $D k k 3$ mRNA expression in mice at postnatal days $1,7,14,16$, and 20 , as well as 8 weeks after birth. (F) Representative immunofluorescence images of $D k k 3$ - $L C h$ mouse kidneys 0 (Ctrl, $n=4)$ and 7 days after UUO $(n=4)$, stained for mCherry (red)/aquaporin 1 (AQP1, green) (upper panel) or mCherry (red)/aquaporin 2 (AQP2, green). (C) Representative images of kidney of PAS-stained kidney sections of $D k 3^{f^{f / f f}}(n=7)$ and $D k k 3^{f / f f} P a \times 8^{\text {Cre }}(n=8)$ mice 0 and 21 days after UUO (scale bars: $100 \mu \mathrm{m})$. (H) Quantification of renal segmental differentiated tubules in PAS-stained kidney sections of WT $(n=4), D k k 3^{-/-}(n=4), D k k 3^{\text {fl/f }}$ $(n=7)$, and $D k k 3^{f / f} P a x 8^{\text {Cre }}(n=8)$ mice 21 days after UUO. (I) Representative images of Masson's trichrome-stained kidney sections of $D k k 3^{f / / f l}(n=9)$ and $D k k 3^{f / f} P a x 8^{\text {cre }}(n=9)$ mice 21 days after UUO (Scale bars: $100 \mu \mathrm{m}$ ). (J) Semiquantitative analysis of renal interstitial fibrosis in Masson's trichrome-stained kidney sections of WT $(n=4), D k k 3^{-1-}(n=4), D k k 3^{f / f l}(n=9)$, and $D k k 3^{f / f l} P a x 8^{\text {Cre }}(n=9)$ mice 21 days after UUO. All data are shown as mean \pm SEM. Statistical analysis performed using Mann-Whitney $U$ test. ${ }^{*} P<0.05$; ${ }^{* *} P<0.01$.

tions in the urine (Figure 6A). In order to corroborate and expand these findings to the human system, we examined DKK3 excretion in the urine of patients with CKD. We analyzed urine samples of 72 young patients, ranging from 6.7 to 19.4 years in age, with different types of CKD (Supplemental Table 1). While DKK3 was absent in the urine of age-matched, healthy children, DKK3 protein was detected in CKD patients with different acquired and genetic glomerular and tubulointerstitial diseases (Figure 6B). DKK3 urine levels of these individuals correlated inversely with the estimated glomerular filtration rate (eGFR) (Figure 6, C and D) but not with proteinuria (Figure 6E). In addition, we prospectively studied a cohort of 36 adult patients with different types of degenerative and immunologic glomerular and tubulointerstitial diseases in a double-blinded fashion. These patients had also undergone renal biopsy (Supplemental Table 2). In such patients, DKK3 urine levels strongly correlated with the grades of TA and IF seen in the 
A

\section{$\mathrm{Dkk3}^{-/-} \mathrm{d} 7$ UUO versus WT d7 UUO}

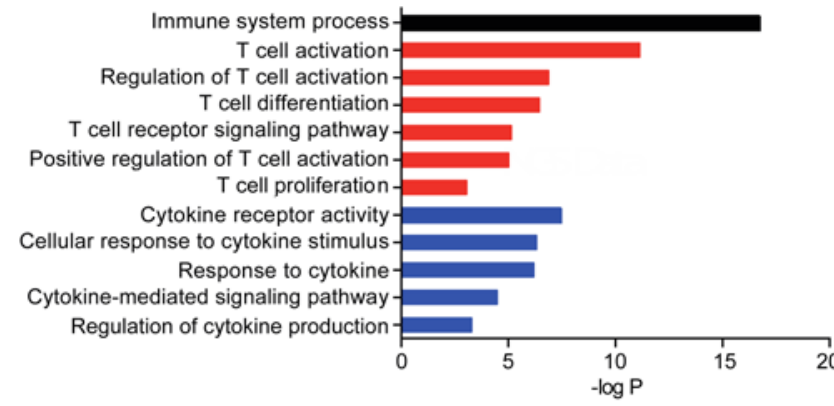

C

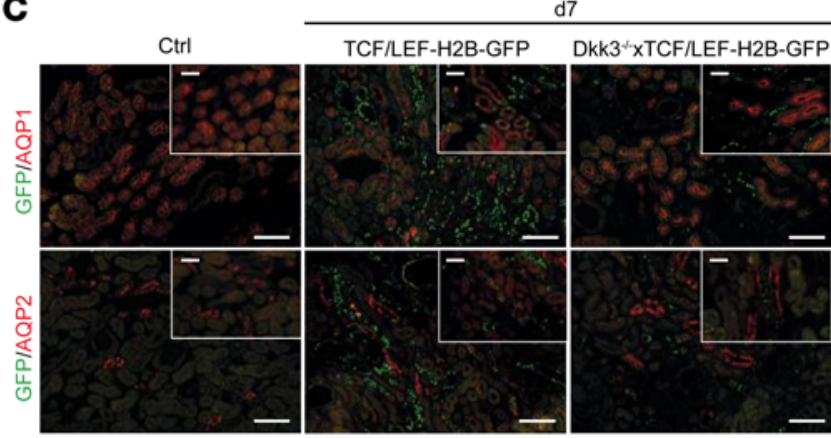

D

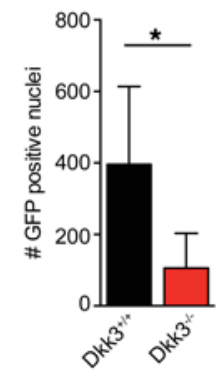

E

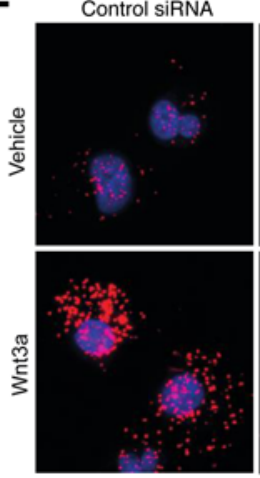

Dkk3 siRNA

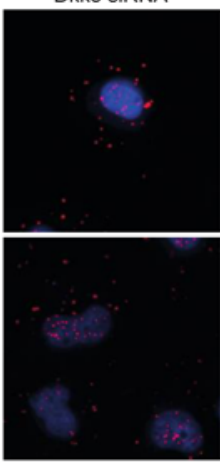

B
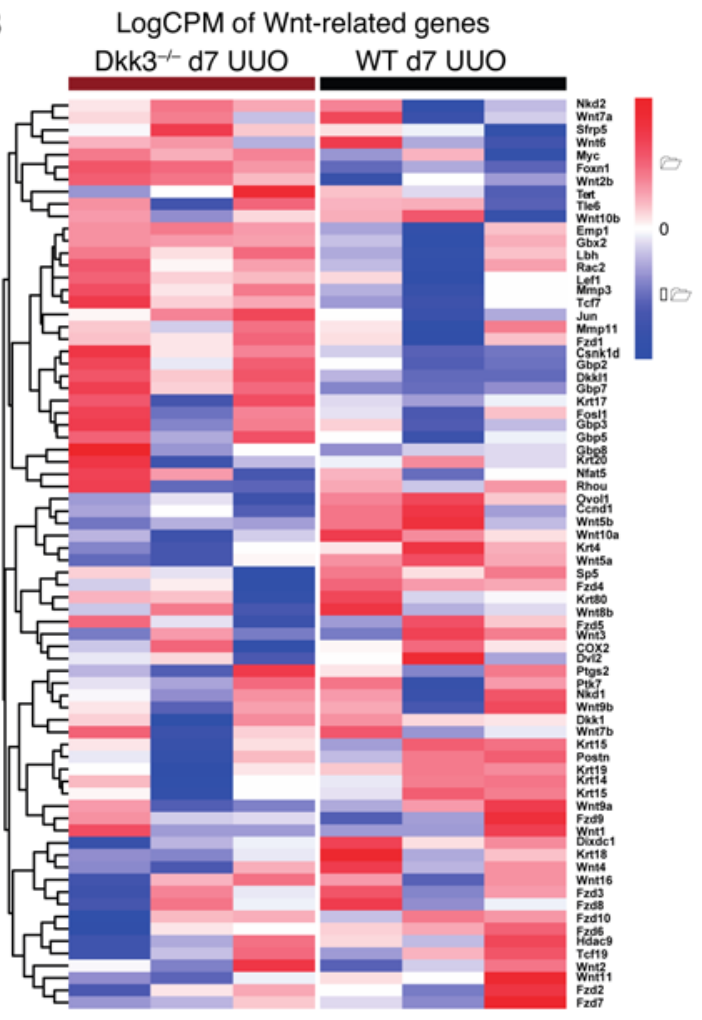

F

G

PLA: DVL/Frizzled DAPI

PLA negative

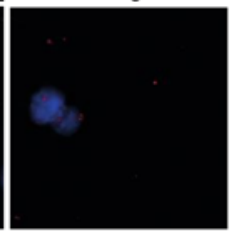

Stress Injury
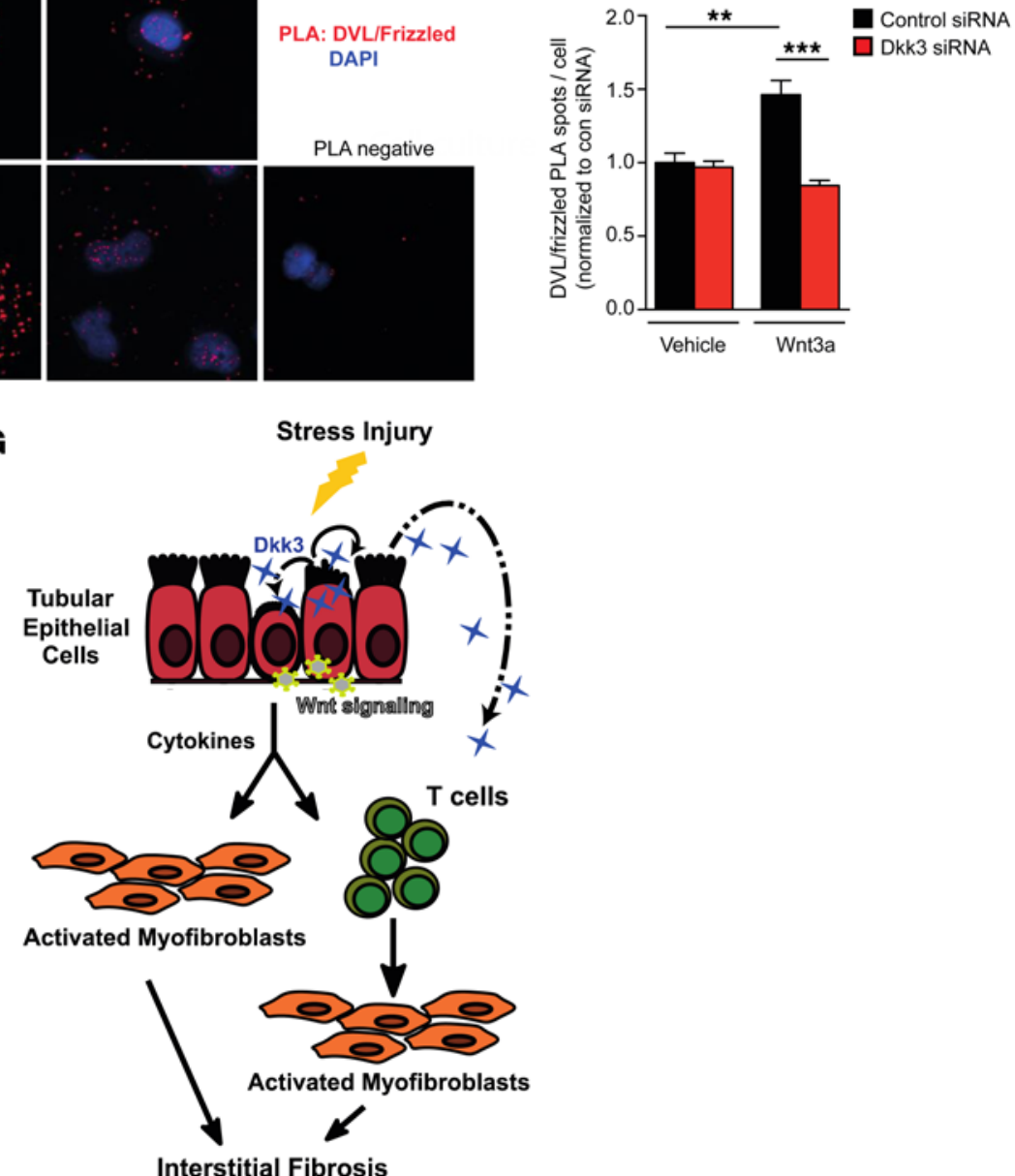
Figure 5. Abrogation of dickkopf 3 (DKКЗ) limits Wnt/ $\beta$-catenin signaling in tubular epithelial cells upon unilateral ureteral obstruction. (A) mRNAseq-based gene ontology analysis of $\mathrm{Dkk3}^{-/-}(n=3)$ versus WT $(n=3)$ mouse kidneys 7 days after unilateral ureteral obstruction (UUO). The graph shows - logP values calculated using Benjamini-Hochberg corrected 2-tailed $t$ test for the enrichment of a specific pathway. (B) Heatmap analysis of the expression of transcripts related to the Wnt signaling in $D k k 3^{-1-}(n=3)$ versus WT $(n=3)$ mouse kidney 7 days after UUO. (C) Representative immunofluorescence images of kidneys of TCF/LEF:H2B-GFP $(n=4)$ and $D k k 3^{-1-} \times T C F / L E F: H 2 B-G F P(n=4)$ mice, stained for GFP (green)/aquaporin 1 (AQP1; red) (upper image) or GFP (green)/ aquaporin 2 (AQP2; red) (lower image) 0 (Ctrl) and 7 days after UUO (scale bars: $50 \mu \mathrm{m}$ ). White dashed line (upper right images) defines the tubular area. (D) Quantification of GFP-positive nuclei in TCF/LEF:H2B-GFP $\left(D k k 3^{+/+}, n=4\right)$ and $D k k 3^{-/-} \times T C F / L E F: H 2 B-G F P$ $\left(D k k^{-1-}, n=4\right)$ mouse kidneys 7 days after UUO. (E) Representative immunofluorescence images of proximity events (red spots) between disheveled (DVL) and frizzled (FZD) in HK2 cells treated either with control siRNA or DKK3 siRNA plus either vehicle or recombinant Wnt3a protein (200 ng/ml) for 1 hour. (F) Quantification of number of proximity events per cell in HK2 cells treated either with control siRNA or DKK3 siRNA, plus either vehicle or recombinant Wnt3a protein $(200 \mathrm{ng} / \mathrm{ml})$ for 1 hour. (G) Schematic illustration of the hypothetical DKK3 function in the development of renal fibrosis. All data are shown as mean \pm SEM. Statistical analysis performed using an unpaired, 2-tailed $t$ test. ${ }^{*} P<0.05 ;{ }^{* *} P<0.01 ;{ }^{* * *} P<0.001$.

renal biopsies (Figure 6, F-J, and Supplemental Table 3). In contrast to the DKK3/atrophy correlation, plasma creatinine concentrations, which are routinely used to evaluate renal function, showed only weak correlation with the extent of TA and IF in these patients (Figure 6, K-N, and Supplemental Table 4). Thus, urinary DKK3 seems to constitute a molecule reflecting the extent of tubular damage and IF in CKD independent of its etiology.

\section{Discussion}

The present study demonstrates a pronounced profibrotic function of DKK3 in the kidney and elucidates mechanisms by which DKK3 promotes renal TA and IF. Intriguingly, we found that DKK3 exhibits two features that are often assumed to exclude each other; it acts as an immunosuppressive and, at the same time, profibrotic molecule, a phenomenon also seen with calcineurin inhibitors.

We provide evidence that DKK3 is expressed in the developing kidney, shut off in adult life, and is neoexpressed in stressed TECs. We found that DKK3 positively regulates Wnt/ $\beta$-catenin signaling in tubular epithelia and engages a profibrotic $\mathrm{T}$ cell response. Its genetic deletion as well as antibody-mediated blockade inhibited the development of TA and IF and improved kidney function in two progressive models of renal fibrosis. Finally, in a prospective patient study, urinary DKK3 protein could be shown to be a robust, noninvasive indicator for chronic tubulointerstitial damage, which determines renal excretory function.

Due to this temporospatial expression pattern, DKK3 seems to be a member of evolutionarily conserved gene clusters, which are active during developmental processes, silenced in homeostasis, and reexpressed under pathologic conditions.

Our results reveal that TECs are the source of DKK3 in the diseased kidney and are, at the same time, the major paracrine/autocrine target of DKK3. Several in vitro studies have reported that DKK3 has the property to either support or suppress canonical Wnt/ $\beta$-catenin signaling, depending on tissue context (24, 25). This cell type- and microenvironment-dependent function has also been proposed for DKK2 (18). Our experiments with two different models of chronic renal disease have now shown, for the first time to our knowledge, that DKK3 can support the activation of Wnt/ $\beta$-catenin signaling in vivo. This is in contrast to its family member DKK1, which was shown to inhibit Wnt signaling and to diminish renal IF (31).

Recently, it has been described that depletion of $\beta$-catenin in TECs had little effect on the severity of renal fibrosis 7 days after UUO. However, pathology at this time point is minimal in WT mice and does not mirror relevant IF (32). In our study, DKK3 depletion resulted in minor phenotypical improvement 7 days after UUO but in significant tissue protection 21 days after renal obstruction. However, these results may also be interpreted such that DKK3 is acting via other signaling pathways, in addition to Wnt.

Administration of recombinant Wnt-pathway inhibitors such as soluble FZD protein (sFRP) (33), Klotho $(34)$, or DKK1 $(35,36)$ have been reported to reduce kidney injury and improve renal function in models of CKD. As antibody-mediated blockade of DKK3 significantly improved renal morphology and function, this might represent a recombinant protein-independent, antibody-based therapeutic approach to arrest progressive CKD.

A chronic, nonresolving inflammation is a hallmark of progressive renal fibrosis (37). T cells are important contributors to this process (38). Therefore, it is intriguing that protection of renal morphology and function in the absence of DKK3 was accompanied by an increased T cell accumulation in the renal interstitium with a bias toward IFN $\gamma$-producing Th1 and Tregs. It has been reported that Th1 cells, and IFN $\gamma$ in particular, can exert a beneficial action in renal fibrosis (39), while Th2 cells and their signature cytokines 
A

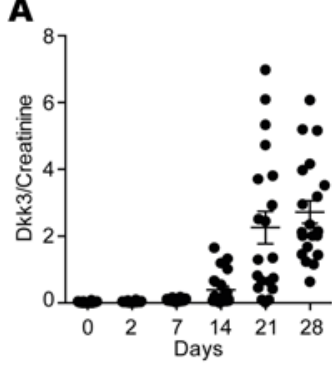

B

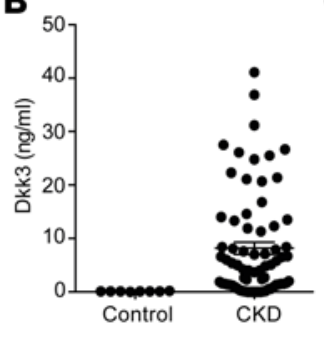

C

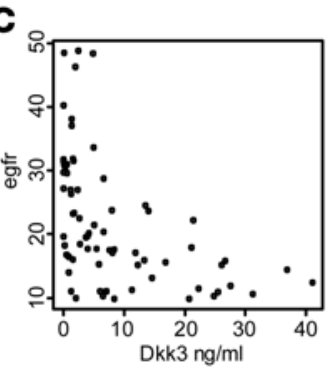

D

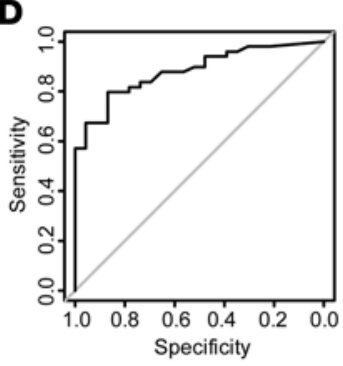

E

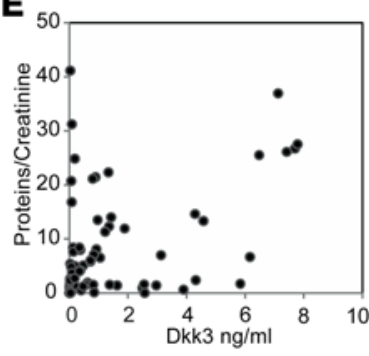

F

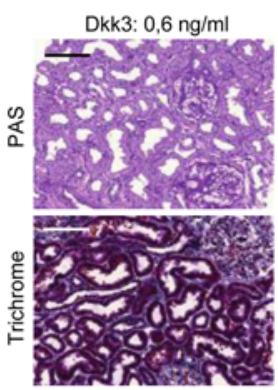

Dkk3: $5,7 \mathrm{ng} / \mathrm{ml}$ Dkk3: $14,4 \mathrm{ng} / \mathrm{ml}$ Dkk3: $24,8 \mathrm{ng} / \mathrm{ml}$ Dkk3: $30,8 \mathrm{ng} / \mathrm{ml}$
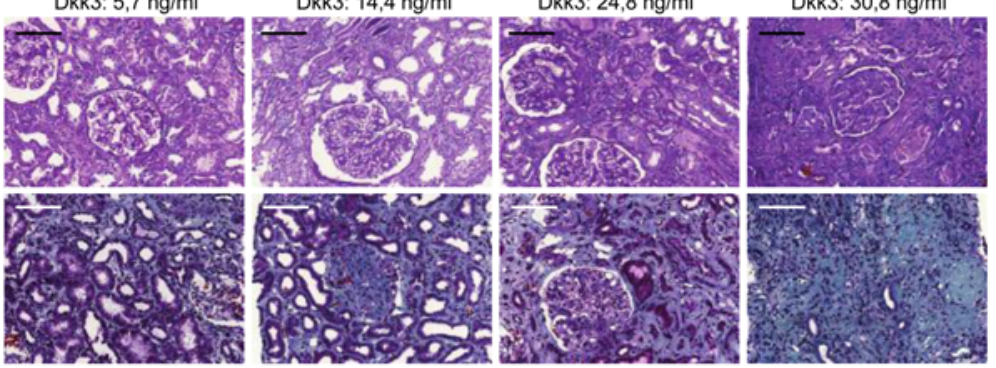

G

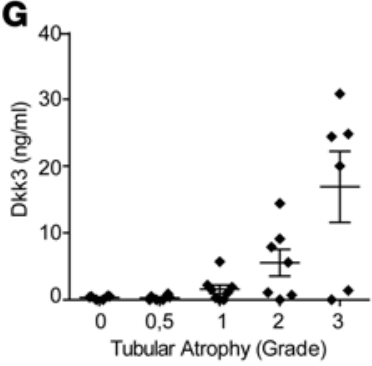

K

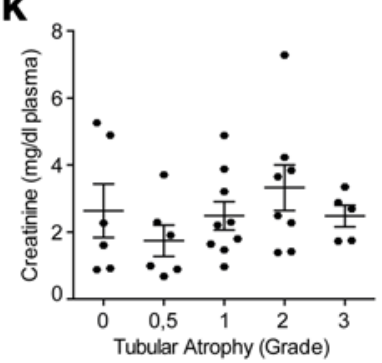

H

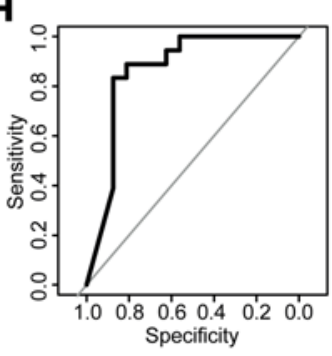

L

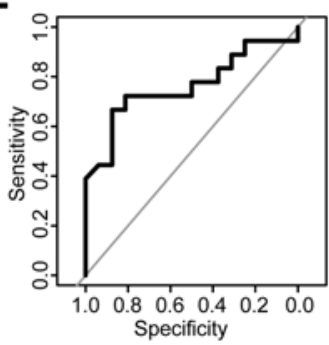

I

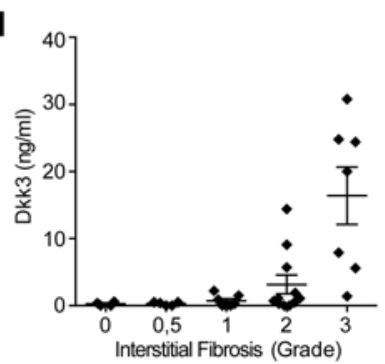

M

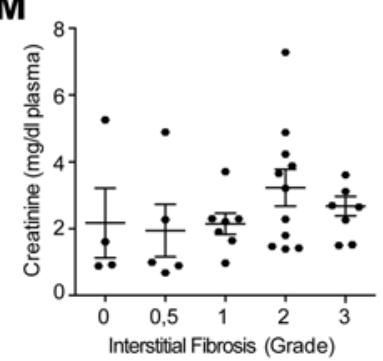

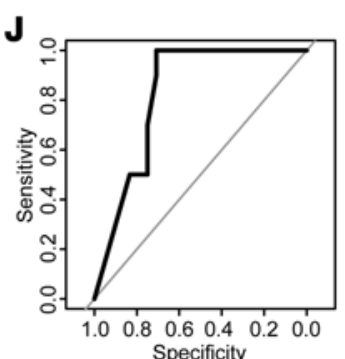

$\mathbf{N}$

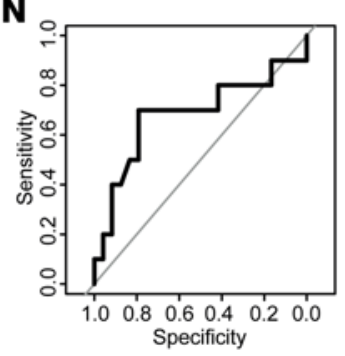

Figure 6. Dickkopf 3 (DKK3) is a biomarker for tubular atrophy and interstitial fibrosis in the urine of patients suffering from progressive CKD. (A) DKK3 protein levels in the urine of mice fed with adenine-enriched diet for $2,7,14,21$, and 28 days $(n=20)$, detected by ELISA. As a control, urine from mice before adenine administration was used. (B) DKK3 protein levels in the urine of healthy, young volunteers (control, $n=8$ ) and CKD-suffering patients $(n=72)$, detected by ELISA. (C) Correlation of DKK3 urine levels and the respective estimated glomerular filtration rate (eGFR) of pediatric patients' cohort samples. (D) Receiver operating characteristic (ROC) curve derived for DKK3, using a threshold of $25 \mathrm{ml} / \mathrm{min} / 1.73 \mathrm{~m}{ }^{2}$ for eGFR and defining the 2 patient groups as eGFR $\leq 25$ = severe disease and eGFR $>25=$ nonsevere disease. AUC $=0.8815$ ( $95 \% \mathrm{Cl}, 0.8056-0.9575$ ). (E) Correlation of DKK3 urine levels and protein/creatinine levels of pediatric patients. (F) Representative images of Periodic acid-Schiff- (PAS-) and trichrome-stained renal biopsies of CKD-affected patients and related urine DKK3 concentration (scale bars: $100 \mu \mathrm{m}$ ). (C) Correlation of DKK3 urine levels and the grade of tubular atrophy (TA) in adult patients' cohort samples. TA grades are defined as $0=0 \%-4.9 \%$ TA; $0.5=5 \%-15 \%$ TA; $1=$ $15.1 \%-33 \%$ TA; $2=33.1 \%-66 \%$ TA; $3=66.1 \%-100 \%$ TA. (H) ROC curve derived for DKK3, using a threshold of $25 \%$ TA and defining the 2 patient groups as TA $\leq 25 \%=$ nonsevere disease and TA $>25 \%=$ severe disease. AUC $=0.8646(95 \% \mathrm{Cl}, 0.7248-1)$. (I) Correlation of DKK3 urine levels and the grade of interstitial fibrosis (IF) in adult patients' cohort samples. IF grades are defined as $0=0 \%-4.9 \%$ IF; $0.5=5 \%-15 \%$ IF; $1=15.1 \%-33 \%$ IF; $2=33.1 \%-66 \%$ IF; $3=66.1 \%-100 \%$ IF. (J) ROC curve derived for DKK3, using a threshold of $25 \%$ IF and defining the 2 patient groups as IF $\leq 25 \%=$ nonsevere disease and IF $>25 \%$ = severe disease. AUC $=0.825$ (95\% Cl, 0.6911-0.9589). (K) Correlation of creatinine plasma levels and the grade of TA in adult patients' cohort samples. The TA grades are defined as in G. (L) ROC curve derived for creatinine, using a threshold of $25 \%$ TA and defining the 2 patient groups as TA $\leq 25 \%=$ nonsevere disease and TA $>25 \%=$ severe disease. AUC $=0.7622$ ( $95 \% \mathrm{Cl}, 0.5939-0.9304)$. (M) Correlation of creatinine plasma levels and the grade of IF in adult patients' cohort samples. The IF grades are defined as in I. (N) ROC curve derived for creatinine, using a threshold of $25 \%$ IF and defining the 2 patient groups as IF $\leq 25 \%=$ nonsevere disease and IF $>25 \%=$ severe disease. $\mathrm{AUC}=0.6812(95 \% \mathrm{Cl}, 0.445-0.9175)$. 
IL-4 and IL-13 have been proposed to be profibrotic (40). Interestingly, this T cell phenotype also recapitulates our previous findings in a mouse model of multiple sclerosis, showing an increased accumulation of IFN $\gamma$-producing $\mathrm{CD}^{+}$and $\mathrm{CD}^{+} \mathrm{T}$ cells within the CNS of DKK3-deficient mice (27). Even though the role of Tregs in kidney fibrosis is still controversial (41), the increase of these cells during genetic deletion or antibody-mediated blockade of DKK3 might contribute to the limitation of an excessive Th1 activity.

The observed $\mathrm{T}$ cell phenotype could, in part, result from a direct impact of DKK3 on T cells. Wnt/ $\beta$ catenin signaling was shown to regulate proliferation and differentiation of $\mathrm{CD}^{+} \mathrm{T}$ cells (42). In addition, the transcription factors TCF1 and LEF have been suggested to promote Th2 differentiation (43) and to repress Th1 as well as Treg development $(44,45)$.

Decreased Wnt signaling in TECs caused by the absence of DKK3 may further impact T cell function. This is indicated by our findings that DKK3 abrogation in tubular cells results in an increased stress-induced production of IL- 6 and IL-8 and thus in a proinflammatory phenotype. These results are in line with studies revealing that Wnt signaling can modulate immune responses via its interconnection with proinflammatory pathways like the $\mathrm{NF} \kappa \mathrm{B}$ signaling cascade (46), which is also essential for stress-triggered cytokine and chemokine expression by TECs (47). For example, $\beta$-catenin activation was shown to inhibit expression of cytokines like TNF $\alpha$ or IL-6 in epithelial cells (48). Moreover, it was recently reported that melanoma-intrinsic Wnt/ $\beta$-catenin signaling regulates $\mathrm{T}$ cell infiltration into the tumor (49), emphasizing that Wnt signaling in parenchymal/tumor cells may have the capacity to directly influence $\mathrm{T}$ cell behavior. To our knowledge, this is the first report describing a Wnt regulator, DKK3, to influence such processes.

Reliable diagnostic tools for the assessment of chronic renal disease and related organ damage are missing. There are several urinary proteins indicating acute renal injury but only a few reflecting the extent of chronic renal damage, which can, up to now, only inaccurately be estimated by plasma creatinine concentration and formula-derived eGFR. Intriguingly, we observed that urinary DKK3 levels in patients with different types of CKD correlated with the degree of renal tissue damage and IF more accurately than plasma creatinine levels. Therefore, we propose that measurement of DKK3 levels in patients' urine may serve as a general indicator for progressive CKD and additionally may provide valuable information concerning the extent of TA and IF, which determines renal excretory function and is an accepted prognostic indicator of $\mathrm{CKD}$

In summary, our studies identify DKK3 as a tubular-derived, immunosuppressive, and profibrotic molecule that maybe a potential therapeutic target as well as a noninvasive marker for the assessment of TA and IF in CKD.

\section{Methods}

Mice. Male, 8- to 12-week-old mice were used for the experiments. C57BL/6 mice were purchased from The Jackson Laboratory. $D k k 3^{-1-}$ and $R a g 2^{-1-}$ mice have been described $(50,51)$. $D k k 3^{-1-}$ mice were crossed to TCF/LEF:H2B-GFP mice (52), which were purchased from The Jackson Laboratory. Mice were maintained under specific pathogen-free conditions at the German Cancer Research Center.

Generation of Dkk3 reporter mice (Dkk3-LCh). Generation of a CB99Luciferase-2A-mCherry construct has been described (53). The CB99Luciferase-2A-mCherry cassette was inserted into the ATG of the Dkk3 gene within a BAC containing the entire Dkk3 gene and its regulatory elements (BAC clone RP23-12M6) (Source BioScience). After removal of the excessive vector, the DNA construct was purified and injected into $\mathrm{C} 57 \mathrm{BL} / 6 \mathrm{~F}_{2}$ oocytes, which were transplanted to super-ovulated C57BL/ 6 females. Founder lines were selected by signal intensity.

Generation of conditional $D k k 3^{-/-}$mice $\left(D k k 3^{f / f t}\right)$. An embryonic stem cell clone containing a floxed allele of DKK3 (clone EPD0642_3_H05) was purchased from The Knockout Mouse Project (KOMP). Dkk3 $3^{\text {flfl }}$ were crossed to Pax $8^{+/ C r e}$ mice (30) in order to obtain a conditional DKK3 KO in renal tubular cells.

UUO. UUO was performed as described previously (54). Briefly, under general isoflurane anesthesia (1.8\%) a low middle-left incision was made on the abdominal side of the mouse, and the left distal ureter was ligated with a 2/0 Mersilene suture. All surgery procedures were performed on a heating pad. Mice were euthanized under general isoflurane anesthesia after either 7 or 21 days.

Adenine nephropathy. Food enriched with adenine (0.25\%, Calbiochem) was administered to mice. The diet was administered for 7 days (early damage) and 28 days (late damage). Afterward, mice were sacrificed.

Renal histology and morphometry. Ligated and contralateral mouse kidney slices were fixed in $4 \%$ formaldehyde in PBS or zinc solution for histological and immunohistological analyses. Tissue slices were snap 
frozen in liquid nitrogen and were stored at $-80^{\circ} \mathrm{C}$. Paraffin-embedded kidney sections $(4 \mu \mathrm{m})$ were cut for quantitative and semiquantitative analysis. Slides were stained with periodic acid-Schiff (PAS) reagent and Masson's trichrome for tubular damage and interstitial scarred area, respectively. Morphometric analysis was performed on PAS sections using a semiautomatic image analyzing system (Leica Q600 Qwin; Leica Microsystems), and the percentage of differentiated proximal tubules was evaluated by examining 18 fields of cortex and inner medulla at a magnification of $\times 20$, obtained after exclusion of glomeruli. The extent of Masson's trichrome staining and of $\alpha$-SMA was assessed as 0 representing no staining detectable, 1 representing mild staining, 2 representing moderate staining, and 3 representing intense staining. A degree-specific damage index was defined as the percentage of fields with the respective degree of injury, and the total staining score index was calculated as the sum of specific damage indices, whereby the index of the fields with degree 1 was multiplied by 1 , that of degree 2 by 2 , and that of degree 3 by 3 . Human biopsies were evaluated for TA and IF defining the grade as follows: $0=0 \%-4.9 \%$ TA (IF); $0.5=5 \%-15 \%$ TA (IF); $1=15.1 \%-33 \%$ TA (IF); $2=33.1 \%-66 \%$ TA (IF); $3=66.1 \%-100 \%$ TA (IF).

$I H C$. For IHC, zinc-fixed tissues were used. The following primary antibodies were taken: anti- $\alpha$-SMA (A2547, 1A4 mouse ascites fluid, Sigma-Aldrich), anti-F4/80 (MCA497, CI:A3-1 rat, 1:100, AbD Serotec), anti-CD3- $\varepsilon$ (SC-1127, M-20 goat, 1:100, Santa Cruz Biotechnology Inc.), and anti-FoxP3 (14-5773, FJK-16s rat, 1:50, eBioscience). Control sections were made by the omission of the primary antibody. The number of positive cells for each kind of marker was counted in 18 high-power fields $(\mathrm{HPF}, \times 40)$ of the tubule-interstitium (12 HPF at the kidney sides, and $6 \mathrm{HPF}$ in the middle to avoid a skew in the results).

Immunofluorescence. Right and left kidneys from mice treated with both UUO (7 and 21 days) and an adenine-rich diet ( 7 and 28 days), and from the same mice without treatment, were fixed in $4 \%$ formalin for 24 hours at room temperature. The tissues were then placed overnight in a PBS solution with $30 \%$ of sucrose at $4^{\circ} \mathrm{C}$. Kidneys were cut in 3 slices, frozen in liquid nitrogen, and stored at $-80^{\circ} \mathrm{C}$. Antibodies against AQP 1 (SC-20810, H-55 rabbit, Santa Cruz Biotechnology Inc.) AQP2 (SC-9882, C-17 goat, 1:800, Santa Cruz Biotechnology Inc.), keratin-18 (61028, Ks 18.04 mouse, 1:50, Progen Biotechnik Gmb), and Fibronectin (A0245, polyclonal rabbit, 1:500, DAKO) were used. DAPI counterstaining was used to detect nuclei.

Antibody treatment. UUO-induced C57BL/6 mice were divided into two groups and received either the anti-DKK3 4.22 monoclonal antibody (28) or the MOPC21 antibody (Bio X Cell; 4666-3/0313 BE0083) as isotype control. In both cases, the first injection was made immediately after surgery with $1.0 \mathrm{mg}$ of the assigned antibody. The related antibody $(0.5 \mathrm{mg})$ was administered every third day until the final day (21 days after UUO), when the animals were sacrificed. Each antibody was i.p. injected.

Flow cytometry. Surface-antigen staining was performed according to standard protocols using the following antibodies: CD3-PerCpCy5.5 (17A2, BioLegend), CD4-PB (RM4-5, BioLegend), CD8-APC-Cy7 (53-6.7, eBioscience), CD25-PE (PC61, BD Biosciences), and CD44-PeCy7 (IM7, BioLegend). For intracellular cytokine staining, lymphocytes were restimulated with $1 \mu \mathrm{g} / \mathrm{ml} \mathrm{PMA} /$ ionomycin in the presence of Golgi Plug (BD Biosciences) for 6 hours. The staining was performed with Cytofix/Cytoperm reagents (BD Biosciences) according to the manufacturer's protocol. TNF-PE/IFN $\gamma$-APC (Mab1/XMG1.2, BD Biosciences) was used. Intracellular transcription factor-staining was performed using a FoxP3 staining buffer set, an anti-FoxP3-APC and an anti-GATA3 antibody (FJK-16s/TWAJ, eBioscience) according to the manufacturer's protocol. Flow cytometry was performed on a FACSCanto with FACS Diva software (BD Biosciences) and analyzed with FlowJo 9.6.

Ex vivo bioluminescence imaging. Mice were i.p. injected with a dose of $150 \mathrm{mg} / \mathrm{kg}$ D-Luciferin (Synchem UG \& Co. KG) dissolved in PBS 5 minutes before they were sacrificed. Subsequently, organs were taken out and incubated in a solution of D-Luciferin in PBS $(1 \mathrm{mg} / \mathrm{ml})$ for 5 minutes at $37^{\circ} \mathrm{C}$. Photographic images and luminescent images, with an exposure time of 5 minutes, were acquired on an IVIS bioluminescence imaging system 100 (PerkinElmer).

Renal and splenic lymphocyte isolation. Kidneys and spleens were mechanically disrupted in 6-well plates and digested for 15 minutes in $5 \mathrm{ml}$ of digestion solution of RPMI medium 1,640/0.5 mg/ml collagenase IA $/ 4.5 \mathrm{k}$ units $/ \mathrm{ml}$ DNase I (all Sigma-Aldrich) at $37^{\circ} \mathrm{C}$ and $5 \% \mathrm{CO}_{2}$. After repetitive pipetting, the organ homogenates were sieved through a $100-\mu \mathrm{m}$ filter (BD Biosciences) and afterward through a $30-\mu \mathrm{m}$ filter (BD Biosciences). Samples were centrifuged at $200 \mathrm{~g}, 37^{\circ} \mathrm{C}$ for 5 minutes, and the supernatant was discarded. Spleen samples were treated for 10 minutes at room temperature with red blood cell lysis buffer and centrifuged at $200 \mathrm{~g}, 37^{\circ} \mathrm{C}$ for 5 minutes. Subsequently, lymphocytes were isolated using CD5 (Ly-1) MicroBeads, MS columns (Miltenyi Biotec) according to manufacturer's protocol. 
Cell culture. HK2 immortalized human proximal tubular cells were obtained from ATCC. Cells were grown in DMEM supplemented with 10\% FCS (Invitrogen), and 1\% penicillin/streptomycin. HK2 cells were plated in 12-well plates (Corning Inc.) at 75,000 cells per well and transfected with siRNA for DKK3 (Invitrogen). A nonsilencing siRNA was used as negative control. LipofectAMINE 2000 (Invitrogen) was used according to manufacturer's recommendations for the transfection procedure. After 48 hours, the cells were harvested and used for RNA isolation and FACS analysis.

$R N A$ isolation and quantitative PCR. Total RNA was extracted from kidneys and cells using Chomczynski and Sacchi's protocol (55). RNA 6000 Nano Chip (Agilent Technologies) was used to check RNA quality. According to the standard protocol, $10 \mu \mathrm{g}$ of total RNA was digested with DNase I. Also, $2 \mu \mathrm{g}$ of total RNA (DNA free) was used for the first-strand cDNA synthesis using SuperScript II Reverse Transcriptase and oligo(dT)12-18 (Invitrogen). Real-time PCR was performed on a LightCycler using LightCyler-FastStart DNA Master SYBR green I kit (Roche Diagnostics).

Next-generation sequencing (RNA-Seq). Samples were prepared as a 10-plex, which was sequenced on 3 lanes. HiSeq 2000 Paired-End 50 bp SR (Illumina) run was used. Total amount of $1 \mu \mathrm{g}$ RNA was taken for each sample. Read counts were TMM normalized and tested for differential expression between groups using the negative binomial regression model implemented in the Bioconductor package edgeR (56). Benjamini-Hochberg $(\mathrm{BH})$ correction of $P$ values was applied to control false discovery rate. Differentially expressed genes were tested for over-representation of GO terms using one-sided hypergeometric tests. $P$ values for GO terms were adjusted for multiple testing using BH correction (57). Read counts were transformed to $\log _{2}$ counts per million (cpm) and scaled for visualization in heatmaps. All analyses were performed with R 3.2. Data have been deposited to the GEO database (GSE75925).

Proximity Ligation Assay (PLA, Duolink, Sigma-Aldrich). Cells were pretreated with control siRNAs or DKK3 siRNAs, and stimulated with vehicle or Wnt3a as described in Figure 5E. After fixation (4\% paraformaldehyde, $4^{\circ} \mathrm{C}, 15$ minutes) and blocking (10\% FCS $/ 0.05 \%$ Triton-X in PBS), the PLA was performed according to the manufacturer's guidelines, using primary DVL- (SC-8025, 3F12 mouse, 1:20, Santa Cruz Biotechnology Inc.) and FZD-4 antibodies (SC-66450, C18, 1:20, Santa Cruz Biotechnology Inc.). In case of close proximity between the two target epitopes (approximately $20-30 \mathrm{~nm}$ ), the PLA probe oligonucleotides are ligated in order to form a template for a subsequent rolling circle amplification step. Subsequently, these amplified sequences are then recognized by fluorescently labeled probes, resulting in fluorescent spots that correspond to the presence of proximity events. Formation of PLA spots was then analyzed by fluorescence microscopy (Keyence), and the number of spots per cell was quantified.

Cytokine measurement. IL- 6 and IL-8 protein concentrations were measured using the BD Cytometric Bead Array system (human IL-6 and human IL-8 Flex Sets) according to the manufacturer's guidelines. Analysis was performed using a FACSCalibur and the FCAP Array software, version 3.0.1 (BD Biosciences).

Generation of a monoclonal mouse anti-human DKK3 antibody. A human DKK3-mouseIgG2b fusion pro-

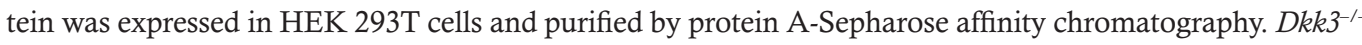
mice were immunized several times with fusion protein, and splenocytes were fused with myeloma cell line X63-Ag8.653. Resulting hybridoma cultures were initially screened by ELISA for reactivity with the fusion protein and subsequently by Western blot using cell lysates derived from human DKK3-expressing tissues. Thus, hybridoma clone huDkk16.1 (IgG1, $\kappa$ isotype) was identified and cloned by limiting dilution.

Mouse urine collection and analysis. Spontaneous urine was collected from C57BL/ 6 mice before and at days $2,7,14,21$, and 28 after the beginning of an adenine-rich diet. Urine was used for DKK3 and creatinine measurements.

Human urine and plasma collection. Urine was collected from pediatric and adult patients and was used for DKK3 and creatinine measurements. Plasma of the respective individuals was used for creatinine determination.

ELISA. A Flexible Assay Plate (BD Biosciences) was coated with anti-mDKK3-4.22 monoclonal antibody (28) $(1 \mu \mathrm{g} / \mathrm{ml})$ or anti-huDkk3-16.1 monoclonal antibody. Biotinylated goat anti-mouse DKK3 antibody (R\&D Systems) biotinylated at a final concentration of $1 \mu \mathrm{g} / \mathrm{ml}$ was used as detection antibody. Streptavidin-Peroxidase (Jackson ImmunoResearch) diluted in PBS-Tween was added for 30 minutes at room temperature. After washing orthophenylene diamine substrate at a concentration of $1 \mathrm{mg} / \mathrm{ml}$ in $100 \mathrm{mM}$ sodium hydrogen, phosphate buffer containing $0.03 \%$ peroxide was applied. The reaction was stopped with $2 \mathrm{M}$ sulphuric acid. Optical densities were analyzed with a Viktor 2 photometer (PerkinElmer) at $490 \mathrm{~nm}$. 
Statistics. Data are shown as mean \pm SEM. If not stated otherwise, statistical analysis was performed using the Mann-Whitney $U$ test. Moreover, for the in vitro experiments, an unpaired, 2-tailed $t$ test was applied. $P<0.05$ was regarded as significant.

Study approval. All animal experiments were approved by the local regulating authorities (Regierungspräsidium Karlsruhe). Urine and plasma collection from young and adult human patients was approved from the ethical committee of the Zentrum für Kinder- und Jugendmedizin, Universitätklinikum Heidelberg, Germany, and from the Ärztekammer des Saarlandes, Saarbrücken, Germany, respectively. Approval for evaluation of respective renal biopsies was awarded by the Ärztekammer des Saarlandes, Saarbrücken.

\section{Author contributions}

GF and MM contributed equally to this work. HJG and BA share senior authorship. HJG and BA provided intellectual input and the conceptual framework and helped to design experiments and to write the manuscript. GF and MM helped to design experiments, performed experiments, collected data, and wrote the manuscript. GHH, GM, FS, and DF provided biological material. DM, AKS, TH, and PJN analyzed data. VN and ZVP performed some experiments and collected data. SP and DF provided intellectual input.

\section{Acknowledgments}

We thank G. Küblbeck, S. Schmitt, S. Prokosch, G. Schmidt, and C. Schmidt for expert technical assistance. We thank C. Niehrs for providing $D k k 3^{-/-}$mice, R. Waldherr for providing human renal biopsy samples, and C. Jäckel and A. Hotz-Wagenblatt for data analysis. This work was supported by the Deutsche Forschungsgemeinschaft (SFB 938 to B. Arnold, H.-J. Gröne, and S. Porubsky; SFB 1118 to H.-J. Gröne).

Address correspondence to: Hermann-Josef Gröne, Department of Cellular and Molecular Pathology, German Cancer Research Center, 69120 Heidelberg, Germany. Phone: 49.6221.424350; E-mail: h.-j.groene@dkfz.de.

1. Wynn TA. Cellular and molecular mechanisms of fibrosis. J Pathol. 2008;214(2):199-210.

2. Liu Y. Cellular and molecular mechanisms of renal fibrosis. Nat Rev Nephrol. 2011;7(12):684-696.

3. Zeisberg M, Neilson EG. Mechanisms of tubulointerstitial fibrosis. J Am Soc Nephrol. 2010;21(11):1819-1834.

4. Bottinger EP. TGF- $\beta$ in renal injury and disease. Semin Nephrol. 2007;27(3):309-320.

5. Jevnikar AM, et al. Stimulated kidney tubular epithelial cells express membrane associated and secreted TNF- $\alpha$. Kidney Int. 1991;40(2):203-211.

6. Schlondorff D. The role of chemokines in the initiation and progression of renal disease. Kidney Int Suppl. 1995;49:S44-S47.

7. Schlondorff D, Nelson PJ, Luckow B, Banas B. Chemokines and renal disease. Kidney Int. 1997;51(3):610-621.

8. Prodjosudjadi W, et al. Production and cytokine-mediated regulation of monocyte chemoattractant protein-1 by human proximal tubular epithelial cells. Kidney Int. 1995;48(5):1477-1486.

9. Tapmeier TT, et al. Pivotal role of $\mathrm{CD}^{+} \mathrm{T}$ cells in renal fibrosis following ureteric obstruction. Kidney Int. 2010;78(4):351-362

10. Duffield JS. Macrophages and immunologic inflammation of the kidney. Semin Nephrol. 2010;30(3):234-254.

11. Glinka A, Wu W, Delius H, Monaghan AP, Blumenstock C, Niehrs C. Dickkopf-1 is a member of a new family of secreted proteins and functions in head induction. Nature. 1998;391(6665):357-362.

12. Krupnik VE, et al. Functional and structural diversity of the human Dickkopf gene family. Gene. 1999;238(2):301-313.

13. Monaghan AP, et al. Dickkopf genes are co-ordinately expressed in mesodermal lineages. Mech Dev. 1999;87(1-2):45-56.

14. Stark K, Vainio S, Vassileva G, McMahon AP. Epithelial transformation of metanephric mesenchyme in the developing kidney regulated by Wnt-4. Nature. 1994;372(6507):679-683.

15. Iglesias DM, et al. Canonical WNT signaling during kidney development. Am J Physiol Renal Physiol. 2007;293(2):F494-F500.

16. Saadi-Kheddouci S, et al. Early development of polycystic kidney disease in transgenic mice expressing an activated mutant of the $\beta$-catenin gene. Oncogene. 2001;20(42):5972-5981.

17. Nelson PJ, von Toerne C, Grone HJ. Wnt-signaling pathways in progressive renal fibrosis. Expert Opin Ther Targets. 2011;15(9):1073-1083.

18. Mao B, Niehrs C. Kremen2 modulates Dickkopf2 activity during Wnt/LRP6 signaling. Gene. 2003;302(1-2):179-183.

19. Niida A, et al. DKK1, a negative regulator of Wnt signaling, is a target of the $\beta$-catenin/TCF pathway. Oncogene. 2004;23(52):8520-8526.

20. Mao B, et al. Kremen proteins are Dickkopf receptors that regulate Wnt/ $\beta$-catenin signaling. Nature. 2002;417(6889):664-667.

21. Niehrs C. Function and biological roles of the Dickkopf family of Wnt modulators. Oncogene. 2006;25(57):7469-7481.

22. Veeck J, et al. Wnt signaling in human breast cancer: expression of the putative Wnt inhibitor Dickkopf-3 (DKK3) is frequently suppressed by promoter hypermethylation in mammary tumours. Breast Cancer Res. 2008;10(5):R82.

23. Caricasole A, et al. Functional characterization of WNT7A signaling in PC12 cells: interaction with A FZD5 x LRP6 receptor complex and modulation by Dickkopf proteins. J Biol Chem. 2003;278(39):37024-37031.

24. Zhu Y, Demidov ON, Goh AM, Virshup DM, Lane DP, Bulavin DV. Phosphatase WIP1 regulates adult neurogenesis and WNT signaling during aging. J Clin Invest. 2014;124(7):3263-3273. 
25. Nakamura RE, Hackam AS. Analysis of Dickkopf3 interactions with Wnt signaling receptors. Growth Factors. 2010;28(4):232-242.

26. Hara K, et al. Blocking of the interaction between Wnt proteins their co-receptors contributes to the anti-tumor effects of adenovirus-mediated DKK3 in glioblastoma. Cancer Lett. 2015;356(2 pt B):496-505.

27. Meister M, et al. Dickkopf-3, a tissue-derived modulator of local T-cell responses. Front Immunol. 2015;6:78

28. Papatriantafyllou M, et al. Dickkopf-3, an immune modulator in peripheral CD8 T-cell tolerance. Proc Natl Acad Sci U S A. 2012;109(5):1631-1636.

29. Meister M, et al. Self-antigen presentation by keratinocytes in the inflamed adult skin modulates T-cell auto-reactivity. J Invest Dermatol. 2015;135(8):1996-2004.

30. Bouchard M, Souabni A, Busslinger M. Tissue-specific expression of cre recombinase from the Pax 8 locus. Genesis. 2004;38(3):105-109.

31. He W, Dai C, Li Y, Zeng G, Monga SP, Liu Y. Wnt/B-catenin signaling promotes renal interstitial fibrosis. J Am Soc Nephrol. 2009;20(4):765-776.

32. Zhou D, Tan RJ, Zhou L, Li Y, Liu Y. Kidney tubular $\beta$-catenin signaling controls interstitial fibroblast fate via epithelial-mesenchymal communication. Sci Rep. 2013;3:1878.

33. Surendran K, Schiavi S, Hruska KA. Wnt-dependent $\beta$-catenin signaling is activated after unilateral ureteral obstruction, and recombinant secreted frizzled-related protein 4 alters the progression of renal fibrosis. J Am Soc Nephrol. 2005;16(8):2373-2384.

34. Satoh M, Nagasu H, Morita Y, Yamaguchi TP, Kanwar YS, Kashihara N. Klotho protects against mouse renal fibrosis by inhibiting Wnt signaling. Am J Physiol Renal Physiol. 2012;303(12):F1641-F1651.

35. Ren S, et al. LRP-6 is a coreceptor for multiple fibrogenic signaling pathways in pericytes and myofibroblasts that are inhibited by DKK-1. Proc Natl Acad Sci U S A. 2013;110(4):1440-1445.

36. He W, Kang YS, Dai C, Liu Y. Blockade of Wnt/ $\beta$-catenin signaling by paricalcitol ameliorates proteinuria and kidney injury. J Am Soc Nephrol. 2011;22(1):90-103.

37. Kurts C, Panzer U, Anders HJ, Rees AJ. The immune system and kidney disease: basic concepts and clinical implications. Nat Rev Immunol. 2013;13(10):738-753.

38. Nikolic-Paterson DJ. CD4+ T cells: a potential player in renal fibrosis. Kidney Int. 2010;78(4):333-335.

39. Oldroyd SD, Thomas GL, Gabbiani G, El Nahas AM. Interferon- $\gamma$ inhibits experimental renal fibrosis. Kidney Int. 1999;56(6):2116-2127.

40. Liu L, et al. CD4 ${ }^{+}$T Lymphocytes, especially Th2 cells, contribute to the progress of renal fibrosis. Am J Nephrol. 2012;36(4):386-396.

41. Gandolfo MT, et al. Foxp3 $3^{+}$regulatory T cells participate in repair of ischemic acute kidney injury. Kidney Int. 2009;76(7):717729.

42. Gattinoni L, et al. Wnt signaling arrests effector T cell differentiation and generates CD8 $8^{+}$memory stem cells. Nat Med. 2009;15(7):808-813.

43. Yu Q, et al. T cell factor 1 initiates the T helper type 2 fate by inducing the transcription factor GATA-3 and repressing interferon-gamma. Nat Immunol. 2009;10(9):992-999.

44. Ma J, Wang R, Fang X, Sun Z. $\beta$-Catenin/TCF-1 pathway in T cell development and differentiation. J Neuroimmune Pharmacol. 2012;7(4):750-762.

45. van Loosdregt J, et al. Canonical Wnt signaling negatively modulates regulatory T cell function. Immunity. 2013;39(2):298-310.

46. Umar S, Sarkar S, Wang Y, Singh P. Functional cross-talk between $\beta$-catenin and NFkB signaling pathways in colonic crypts of mice in response to progastrin. J Biol Chem. 2009;284(33):22274-22284.

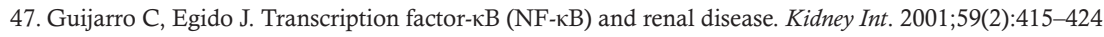

48. Silva-Garcia O, Valdez-Alarcon JJ, Baizabal-Aguirre VM. The Wnt/ $\beta$-catenin signaling pathway controls the inflammatory response in infections caused by pathogenic bacteria. Mediators Inflamm. 2014;2014:310183.

49. Spranger S, Bao R, Gajewski TF. Melanoma-intrinsic $\beta$-catenin signaling prevents anti-tumour immunity. Nature. 2015;523(7559):231-235.

50. Barrantes Idel B, et al. Generation and characterization of dickkopf3 mutant mice. Mol Cell Biol. 2006;26(6):2317-2326.

51. Shinkai Y, et al. RAG-2-deficient mice lack mature lymphocytes owing to inability to initiate V(D)J rearrangement. Cell. 1992;68(5):855-867.

52. Ferrer-Vaquer A, Piliszek A, Tian G, Aho RJ, Dufort D, Hadjantonakis AK. A sensitive and bright single-cell resolution live imaging reporter of Wnt/ss-catenin signaling in the mouse. BMC Dev Biol. 2010;10:121.

53. Miloud T, Henrich C, Hammerling GJ. Quantitative comparison of click beetle and firefly luciferases for in vivo bioluminescence imaging. J Biomed Opt. 2007;12(5):054018.

54. Vielhauer V, et al. Obstructive nephropathy in the mouse: progressive fibrosis correlates with tubulointerstitial chemokine expression and accumulation of CC chemokine receptor 2- and 5-positive leukocytes. J Am Soc Nephrol. 2001;12(6):1173-1187.

55. Chomczynski P, Sacchi N. Single-step method of RNA isolation by acid guanidinium thiocyanate-phenol-chloroform extraction. Anal Biochem. 1987;162(1):156-159.

56. Robinson MD, McCarthy DJ, Smyth GK. edgeR: a Bioconductor package for differential expression analysis of digital gene expression data. Bioinformatics. 2010;26(1):139-140.

57. Fang H, Gough J. The 'dnet' approach promotes emerging research on cancer patient survival. Genome Med. 2014;6(8):64. 